\title{
RNA-Sequencing Data Reveal a Prognostic Four-IncRNA-Based Risk Score for Bladder Urothelial Carcinoma: An in Silico Update
}

\author{
Rong-Quan He $\mathrm{e}^{\mathrm{a}, \mathrm{b}, \mathrm{c}}$ Zhi-Guang Huang ${ }^{\mathrm{b}, \mathrm{c}}$ Tian-Yu Li ${ }^{\mathrm{d}} \quad$ Yan-Ping Wei ${ }^{\mathrm{b}}$ \\ Gang Chen ${ }^{b, c, e} \quad$ Xing-Gu Lin ${ }^{b, c}$ Qiu-Yan Wang ${ }^{a, b, c}$
}

aDepartment of Biochemistry and Molecular Biology, Guangxi Medical University, Nanning, ${ }^{b} C e n t e r$ for Genomic and Personalized Medicine, Nanning, 'Guangxi Collaborative Innovation Center for Genomic and Personalized Medicine, Nanning, dDepartment of Urology, First Affiliated Hospital of Guangxi Medical University, Nanning, e'Department of Pathology, First Affiliated Hospital of Guangxi Medical University, Nanning, China

\section{Key Words}

LncRNA • The Cancer Genome Atlas (TCGA) • Bladder urothelial cancer (BLCA) • Prognosis

\begin{abstract}
Background/Aims: Current practical advances in high-throughput data technologies including RNA-sequencing have led to the identification of long non-coding RNAs (IncRNAs) for potential clinical application against bladder urothelial cancer (BLCA). However, most previous studies focused on the clinical value of individual IncRNAs, which has limited the potential for future clinical application. Methods: In this study, RNA-sequencing data of IncRNAs was downloaded from The Cancer Genome Atlas database. Risk score was constructed based on survivalassociated IncRNAs identified using differential expression analysis as well as univariate and multivariate Cox proportional hazards regression analysis. Receiver operating characteristic and Kaplan-Meier curve analyses were employed to evaluate the clinical and prognostic value of risk scores. Bioinformatics analyses were used to investigate the potential mechanisms of newly identified IncRNAs. Results: Among 2,127 differentially expressed IncRNAs (DELs), four new IncRNAs (AC145124.1, AC010168.2, MIR200CHG, and AC098613.1) showed valuable prognostic effects in BLCA patients. More importantly, the four-DEL-based risk score had the potential to become an independent marker for the survival status prediction of BLCA patients. Distinct co-expressed genes and signaling pathways were identified when BLCA was categorized into low- and high-risk groups. Furthermore, a protein-coding gene, HIST4H4 was found only $68 \mathrm{bp}$ from the AC010168.2 DEL. HIST4H4 expression level was evidently upregulated and positively correlated with AC010168.2 in BLCA patients. Conclusion: This in silico investigation pioneers the future investigation of the utility of prognostic IncRNAs for BLCA.

R.-Q. He and Z.-G. Huang contributed equally to this work.

Rong-Quan He

and Qiu-Yan Wang

Department of Biochemistry and Molecular Biology, Guangxi Medical University

22 Shuangyong Road, Nanning 530021, Guangxi Zhuang Autonomous Region (China)

Tel. 86-0771 5641040, E-Mail herongquan@gxmu.edu.cn; qiuyanwang510@yahoo.com
\end{abstract}


Introduction

Bladder urothelial cancer (BLCA) is the fourth most common cancer in males and the eleventh most common cancer in females, with more than 430, 000 cases worldwide every year [1]. According to the latest epidemiological data of bladder cancer in the United States, there were an estimated 79, 030 new cases in 2017, including 60,490 males and 18, 540 females; 16, 870 patients died, comprising 12, 240 males and 4, 630 females [2]. The incidence and mortality of male patients with BLCA was respectively 4.1 and 3.5 times higher than those of females based on data from 2009 to 2013 [2]. In China, BLCA was the thirteenth most common cancer with a total number of 80, 500 cases in 2015 [3]. The mechanisms of BLCA however remain largely unclear, which has led to the paucity of specific biomarkers for screening, surveillance, or prognostic stratification of BLCA [4-10]. Hence, there is an urgent need to identify new reliable biomarkers related to prognosis.

Long non-coding RNAs (lncRNAs) are a class of RNAs lacking protein-coding function which were initially regarded as "junk RNA" $[11,12]$. It has been experimentally validated that IncRNAs are involved in the etiology and pathogenesis of various cancers, including BLCA [13-20]. The recent advances in high-throughput data technologies, including RNAsequencing and microarrays, has guided the identification of lncRNAs for prospective clinical application in BLCA [12, 21-23]. However, most previous studies focused on the clinical value of individual lncRNAs, which has limited potential for future clinical application. The Cancer Genome Atlas (TCGA) database provides valuable lncRNA data from multiple cancer types, and so it is possible to analyze and discover new lncRNA candidates for the prognostic prediction in BLCA. Previously, Bao et al. [24] reported that a four-lncRNA signature was an independent marker for predicting outcomes in patients with BLCA based on TCGA data acquired in May, 2016. Since then, TCGA data and annotation of IncRNAs have been updated significantly. No differentially expressed IncRNAs (DELs) in BLCA were mentioned by Bao et al. [24]. Moreover, no additional datasets have been used to validate their previous finding either. Therefore, in the current study, DELs were first analyzed from RNA-sequencing data. Next, a prognostic four DEL-based risk score was built up and we further investigated the potential mechanism of these lncRNAs using bioinformatical methodologies (Fig. 1).

\section{Materials and Methods}

\section{Study population}

RNA-sequencing reads per kilobase million (RPKM) data of patients with BLCA were obtained from TCGA $[12,25]$. The cohort contained 433 samples, including 414 tumor tissue samples and 19 non-tumor adjacent bladder tissue samples. Clinicopathological parameters were also downloaded from TCGA, such as person neoplasm cancer status, successful outcome of primary therapy, pathologic $\mathrm{T}$ stage, pathologic $\mathrm{N}$ stage, pathologic M stage, pathologic stage, and clinical T stage. After excluding samples without expression data or clinical information, 405 cases and 19 controls remained. Because the dataset was downloaded from TCGA, there was no need to obtain further approval from an ethics committee. The human subject protection and data access policies from TCGA were duly followed when data were processed. 


\section{Cellular Physiology Cell Physiol Biochem 2018;50:1474-1495 \begin{tabular}{ll|l} 
and Biochemistry Published online: 26 October 2018 & $\begin{array}{l}\text { @ } 2018 \text { The Author(s). Published by S. Karger AG, Basel } \\
\text { www.karger.com/cpb }\end{array}$
\end{tabular}}

He et al.: a Prognostic Four-IncRNA-Based Risk Score For BLCA

Screening of DELs in BLCA based on TCGA data

The RNA-seq dataset from TCGA contained 60483 mRNAs and included lncRNA expression data. Dataset was annotated by using GENCODE version GRCh38 (http://www.gencodegenes.org/) [26] and 13918 lncRNAs were identified. Next, the expression data of these 13918 lncRNAs were used for differential expression analysis with the R package edgeR $[27,28]$. Genes were selected with absolute log2 (Fold Change) $>1$ and adjusted false discovery rate $P$ values $<0.05$, after comparing expression data between tumor tissue and adjacent non-tumor bladder tissue. DELs were excluded for the next step of analysis if their expression data were missing for more than $10 \%$ of the samples [28-30].

Construction of a prognostic risk score with DELS

The expression data of each DEL was normalized by using log2 transformation for further analysis. DELs strongly associated with overall survival (OS), with P values lower than 0.05 , were identified by univariate Cox proportional hazards regression analysis using the R "survival" package. Next, the prognostic significance of the identified DELs was examined via multivariate Cox proportional hazards regression using SPSS version 22.0 (IBM Corp., Armonk, NY). We constructed a prognostic risk score of DEL signatures for predicting OS according to the summation of the expression data of prognostic DELs multiplied by the regression coefficient from the multivariate Cox regression model $(\beta)$, described by the formula below:

$$
\text { Risk score }=\sum_{i=1}^{n}(E X P i * \beta i)
$$

BLCA patients were then categorized into low- and high-risk groups based on the median of the newly calculated prognostic risk score [28, 29, 31, 32]. The whole process is presented in Fig. 2.

Moreover, univariate and multivariate Cox proportional hazards regression analyses were carried out to estimate the influence of clinical parameters and the risk score on the OS of BLCA patients. Hazard ratios (HRs) and 95\% confidence intervals (CIs) were also determined. The predictive analysis of patient outcome was carried out using the receiver operating characteristic (ROC) curve based on the risk scores. KaplanMeier analysis was applied to evaluate the different survival rates for BLCA patients with low- or high-risk scores, and a two-sided log-rank test was utilized to evaluate the differences between the low- and high-risk groups by using SPSS 22.0 software.

Validation of prognostic DELs using other datasets

To verify the expression of the prognostic DELs, DEL expression data from Gene Expression Omnibus (GEO) and ArrayExpress were downloaded and analyzed. The following search terms used were used: (bladder OR urothelial OR urinary OR urogenital) AND (cancer OR carcinoma OR tumor OR neoplasm* OR malignant*) AND (lncRNA OR IncRNAs). Expression data of each DEL from both normal and tumor tissue were extracted and compared using Student's $t$ test with SPSS software.

We performed a meta-analysis to further confirm the expression level of DELs in BLCA tissue. The pooled standard mean difference (SMD) with 95\% CI and publication bias based on TCGA and GEO data were calculated using Stata version 12.0 (StataCorp, College Station, TX). A

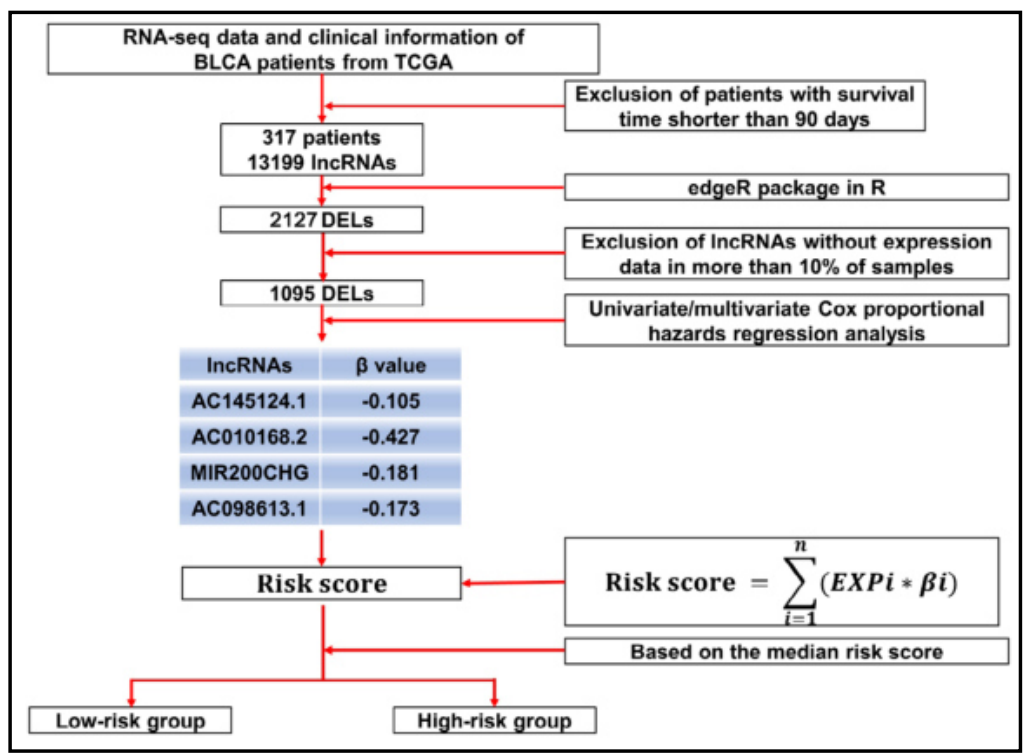

Fig. 2. Procedure for risk score construction. Note: DELs, differentially expressed IncRNAs; BLCA, bladder urothelial carcinoma; TCGA, the Cancer Genome Atlas; $\beta$, regression coefficient. 


\section{Cellular Physiology Cell Physiol Biochem 2018;50:1474-1495 and Biochemistry Published online:26 October 2018 \begin{tabular}{l|l} 
DO 2018 The Author(s). Published by S. Karger AG, Basel \\
www.karger.com/cpb
\end{tabular}}

He et al.: a Prognostic Four-IncRNA-Based Risk Score For BLCA

Begg's funnel plot was also generated.

In addition, we assessed the abundance of the prognostic DELs in other types of tumors, using expression data for the DELs downloaded from Gene Expression Profiling Interactive Analysis (GEPIA, http://gepia.cancer-pku.cn/) to compare of IncRNA expression levels between non-tumor and tumor tissue.

Functional analysis of the selected prognostic DELS

Genes that were co-expressed with the prognostic DELs were obtained from the Multi-Experiment Matrix (MEM) database (http://biit.cs.ut.ee/mem/index.cgi) [33]. Cytoscape and STRING (https://string$\mathrm{db} . o r g /$ ) were also utilized to visualize the prognostic DELs and their corresponding co-expressed genes as a network [34-38].

Potential mechanisms of differentially expressed genes (DEGs) of low- and high-risk groups

As described above, R package edgeR was used to identify DEGs from the low- and high-risk groups, respectively, based on the differential expression analysis between gene expressions of BLCA tissue samples from both groups. Gene Ontology (GO), Kyoto Encyclopedia of Genes and Genomes (KEGG) and proteinprotein interaction (PPI) analyses were carried out to complement the functional enrichment analyses of DEGs using the online software DAVID (https://david.ncifcrf.gov/home.jsp, version 6.8). GO enrichment plot and Disease Ontology analysis were performed with the R packages GOplot and DOSE, respectively.

Relationships between DELs and adjacent protein-coding genes

We then evaluated the relationships between the DELs and adjacent protein-coding genes. We identified these adjacent protein-coding genes using the Ensemble Genome Browser. and then downloaded their expression levels, presented as RPKM values, from TCGA. Differential expression analysis of the adjacent protein-coding genes was then performed. Putative relationships between the DELs and their adjacent protein-coding genes were analyzed by using Pearson's correlation coefficient. ROC curve analysis of adjacent protein-coding genes was performed based on TCGA data. All the above statistical analyses were carried out with SPSS software. Statistical significance was confirmed at a P value less than 0.05. Protein expression data of these genes were further subjected to validation using The Human Protein Atlas (THPA; https://www.proteinatlas.org/) [39]; immunohistochemistry was performed on tissue microarrays for normal bladder and BLCA tissue.

In-house verification of the expression levels of the four IncRNAs and HIST4H4 based on cell lines and BLCA tissue

To validate the results from big data mining based on the TCGA and GEO datasets, RT-qPCR was utilized to measure the expression levels of AC145124.1, AC010168.2, MIR200CHG, AC098613.1, and HIST4H4 in four BLCA cell lines, including 5637, J82, T24, SW780, and a cell line SV-HUC-1, as well as 15 BLCA tissue samples. These tissue samples were obtained from the First Affiliated Hospital of Guangxi Medical University, the ethics committee of which approved this present study. Written informed consent was obtained from the participants or their family. Total RNA was extracted from cell and tissue samples with total RNA miniprep kit (Magen, R4310-02) and then reversed transcribed into cDNA with the cDNA Synthesis Kit (6110A; Takara, Dalian, China). GAPDH was used as the internal reference. Primers used are as follows: AC145124.1, Forward-5' - GGCTAACGTAGGCACTCTCG-3', Reverse-5'- CACTTCTTCCACGAGCACCT-3'; AC010168.2, Forward-5' - TTGGTGTGCAGTTTGGTGTA-3', Reverse-5'- TAAGTGGTCTGCCCATGTTT-3'; MIR200CHG, Forward-5' - CTGACCAACAACCTCTGACCTTCA-3', Reverse-5' - AGGCAGCCATTTTGTCTCCTTC-3'; AC098613.1, Forward-5' - CAGGGAGGAAGGTATGTGGT-3', Reverse-5'- ATCATGTAGGTGCAGGCAGA-3'; HIST4H4, Forward-5'- ATGTGGTGTACGCGCTGAAA-3', Reverse-5'- CGCCAAGTCTAATCCCGAGC-3'; GAPDH, Forward-5' - CTCCAAAATCAAGTGGGGCG-3', Reverse-5' - TGGTTCACACCCATGACGAA-3'. Relative expression was determined by using the formula: Relative expression $=2^{-(\mathrm{Cq}}{ }_{\text {gene }}{ }^{-\mathrm{Cq}_{\mathrm{GAPDH}}}{ }^{\mathrm{f}}[40,41]$. Analysis of the correlation between AC010168.2 and HIST4H4 expression was performed using SPSS 22.0 software. All statistical tests in the study were two-sided, and a P value less than 0.05 was considered significant.

\section{Results}

DELs in BLCA compared to para-cancerous tissue

After the excluding samples from patients with a survival time of less than 90 days, 317 BLCA cases and 14 controls remained. A total of 13, 199 lncRNAs were annotated with GENCODE (http://www.gencodegenes.org/) and analysis of differentially expressed 


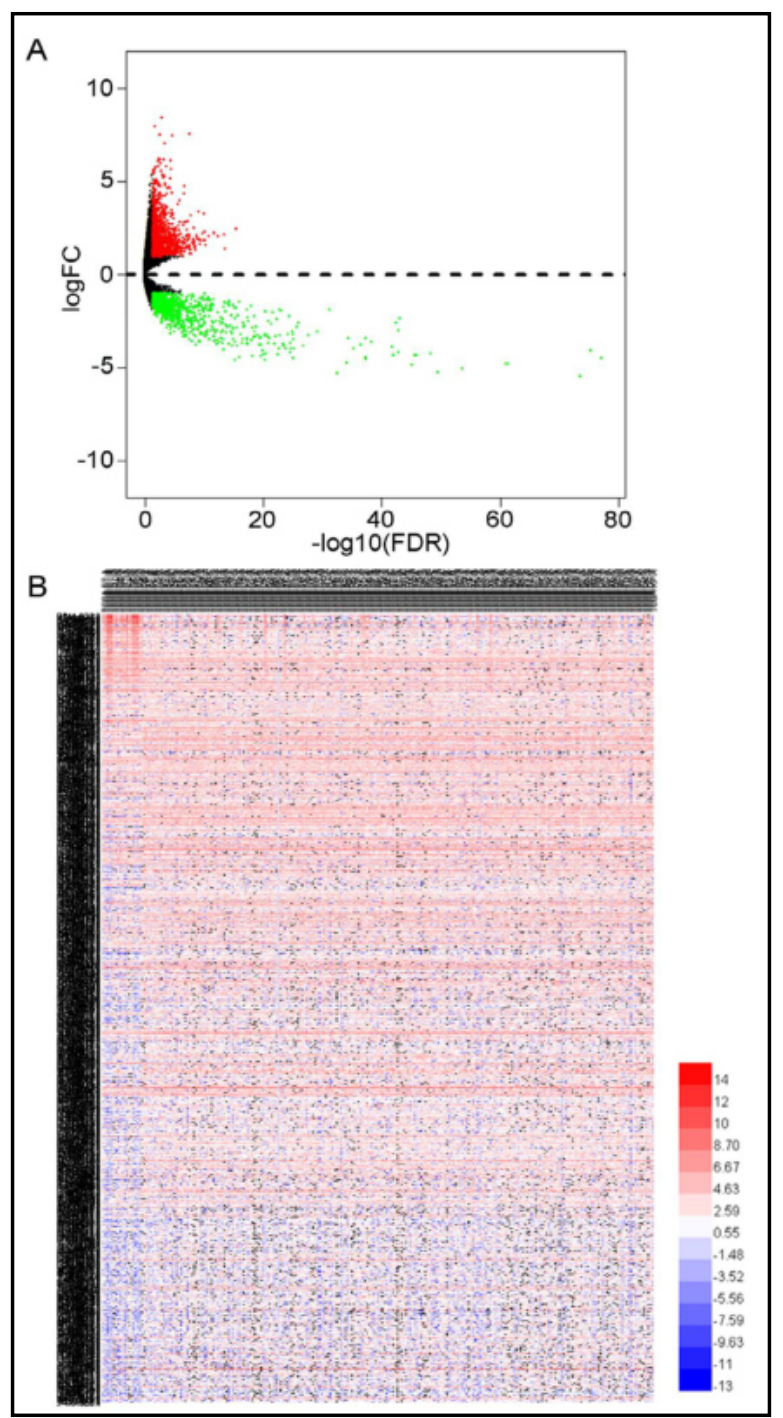

Fig. 3. Differentially expressed IncRNAs (DELs) in bladder urothelial carcinoma (BLCA). (A) Volcano plot of DELs calculated using the edgeR package; (B) heatmap of DELs. Note: FC, fold change; FDR, false discovery rate.
Table 1. Prognostic significance of differentially expressed IncRNAs (DELs) from univariate Cox proportional hazards regression analysis $(\mathrm{P}<0.001)$. These 21 DELs, each with $\mathrm{P}<0.001$, were chosen to perform a multivariate Cox proportional hazards regression analysis. Note: HR, Hazard Ratio

\begin{tabular}{lcccc}
\hline Gene Name & HR & Lower & Higher & P value \\
\hline AC145124.1 & 0.877 & 0.816 & 0.943 & $<0.001$ \\
RNF139-AS1 & 0.602 & 0.455 & 0.796 & $<0.001$ \\
AC011503.2 & 0.807 & 0.730 & 0.893 & $<0.001$ \\
AC010331.1 & 0.709 & 0.593 & 0.848 & $<0.001$ \\
MAFG-AS1 & 1.461 & 1.226 & 1.741 & $<0.001$ \\
AC006128.1 & 0.686 & 0.555 & 0.847 & $<0.001$ \\
U47924.1 & 0.870 & 0.815 & 0.929 & $<0.001$ \\
AC067930.5 & 0.850 & 0.777 & 0.930 & $<0.001$ \\
AL078587.1 & 0.879 & 0.819 & 0.943 & $<0.001$ \\
AC013731.1 & 0.775 & 0.688 & 0.874 & $<0.001$ \\
AC010168.2 & 0.578 & 0.474 & 0.706 & $<0.001$ \\
TMEM72-AS1 & 0.888 & 0.832 & 0.947 & $<0.001$ \\
AC114488.1 & 0.838 & 0.773 & 0.909 & $<0.001$ \\
AL138831.3 & 0.782 & 0.683 & 0.896 & $<0.001$ \\
AL158152.1 & 0.870 & 0.808 & 0.936 & $<0.001$ \\
MIR200CHG & 0.872 & 0.812 & 0.937 & $<0.001$ \\
AC008875.1 & 0.785 & 0.686 & 0.897 & $<0.001$ \\
AC098613.1 & 0.860 & 0.799 & 0.925 & $<0.001$ \\
AC005841.1 & 0.884 & 0.826 & 0.945 & $<0.001$ \\
AL390719.2 & 0.889 & 0.833 & 0.949 & $<0.001$ \\
LIPC-AS1 & 0.868 & 0.820 & 0.920 & $<0.001$ \\
\hline
\end{tabular}

Table 2. Information on the 4 DELs from multivariate Cox proportional hazards regression analysis. Abbreviations: $\beta$, regression coefficient; SE, standard error; HR, Hazard Ratio

\begin{tabular}{|c|c|c|c|c|c|c|c|c|}
\hline & & & & & & & \multicolumn{2}{|c|}{$95.0 \% \mathrm{CI}$ for $\mathrm{HR}$} \\
\hline Gene symbol & Ensemble ID & Location & $\beta$ & SE & $\mathrm{P}$ value & HR & Lower & Upper \\
\hline AC145124.1 & ENSG00000255495 & Chromosome 8: 12,194,467-12,196,280(+) & -0.105 & 0.037 & 0.004 & 0.900 & 0.838 & 0.967 \\
\hline AC010168.2 & ENSG00000261324 & Chromosome 12: 14,762,504-14,767,931(-) & -0.427 & 0.146 & 0.003 & 0.652 & 0.490 & 0.869 \\
\hline MIR200CHG & ENSG00000257084 & Chromosome 12: 6,963,246-6,964,447(+) & -0.181 & 0.057 & 0.002 & 0.835 & 0.746 & 0.934 \\
\hline AC098613.1 & ENSG00000223552 & Chromosome 3: 46,364,955-46,407,059(-) & -0.173 & 0.051 & $<0.001$ & 0.841 & 0.761 & 0.929 \\
\hline
\end{tabular}

IncRNAs between BLCA cases and controls was performed. The edgeR package identified 2, 127 DELs. After excluding DELs with no expression detected in more than $10 \%$ of the samples, 1, 095 DELs remained for prognostic signature construction (Fig. 3).

\section{Construction of the prognostic risk score based on DELs}

Univariate Cox proportional hazards regression analysis unveiled that 21 DELs among the 1, 095 DELs had prominent prognostic values $(\mathrm{P}<0.001)$, which were then used to 


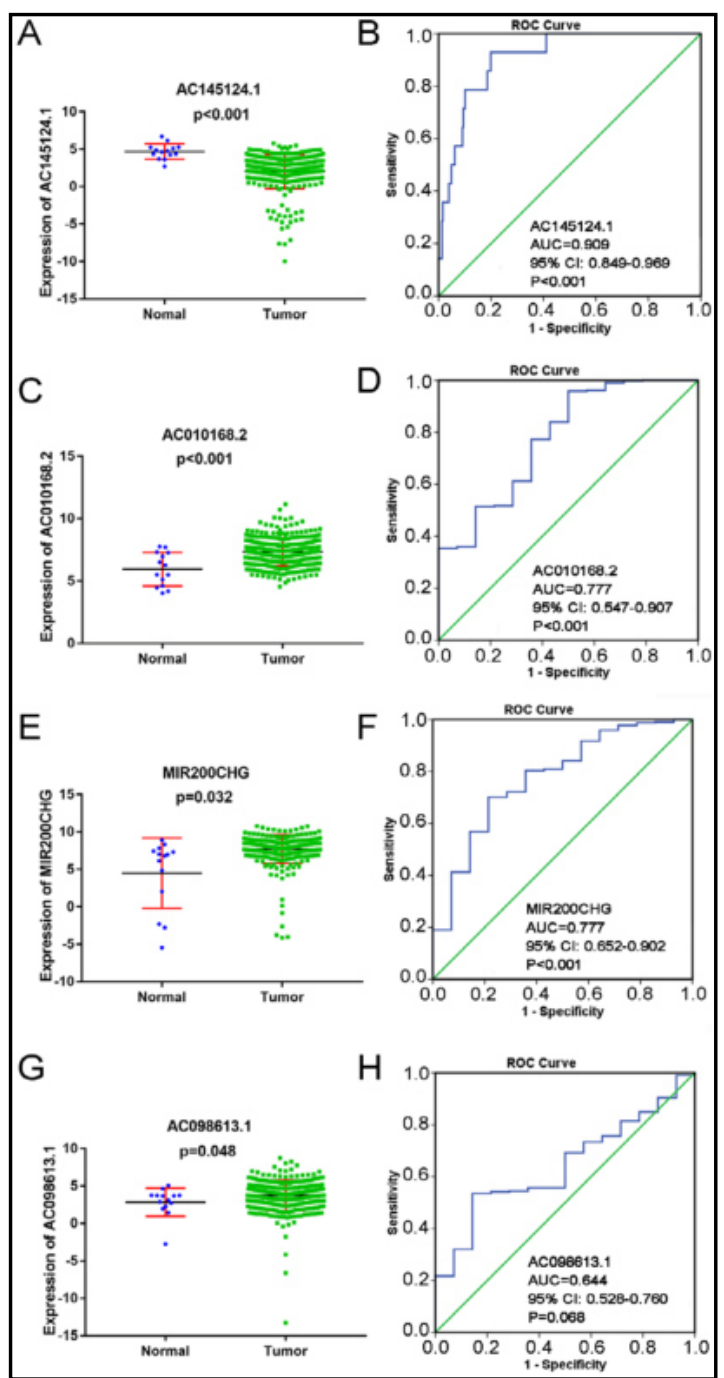

Fig. 4. Expression and clinical value of four differentially expressed IncRNAs (DELs) in bladder urothelial carcinoma (BLCA). (A) Expression of AC145124.1; (B) Receiver operating characteristic (ROC) curve of AC145124.1; (C) Expression of AC010168.2; (D) ROC curve of AC010168.2; (E) Expression of MIR200CHG; (F) ROC curve of MIR200CHG; (G) Expression of AC098613.1; (H) ROC curve of AC098613.1. Patients with BLCA were separated into low and high expression groups based on the median DEL expression level. Note: AUC, area under curve.

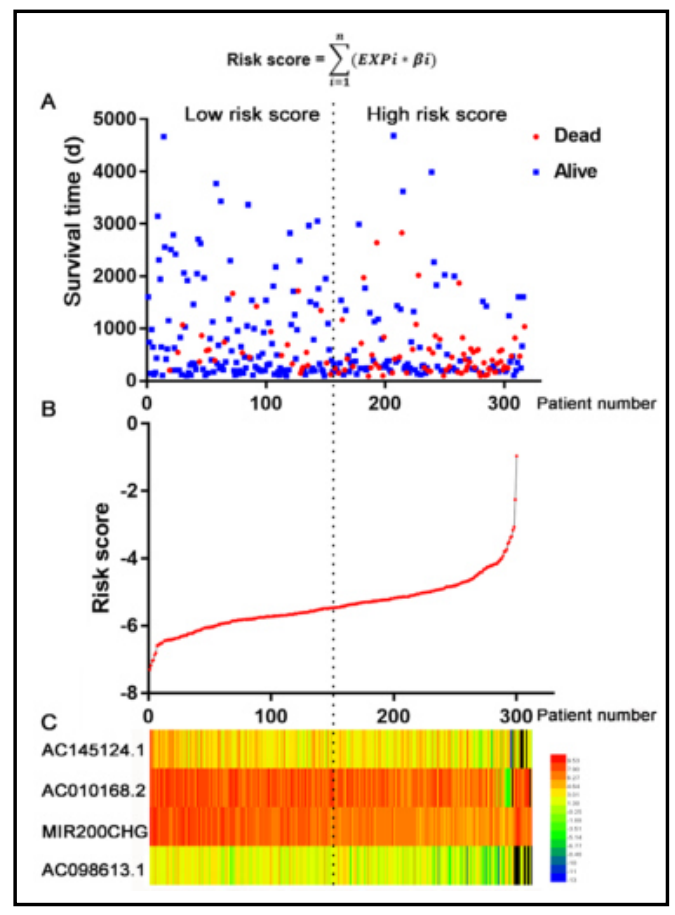

Fig. 5. Analysis of risk score according to the four differentially expressed IncRNAs of BLCA. (A) Survival status of BLCA patients and survival time based on risk score; (B) risk score curve; (C) expression level of four selected DELs represented as a heatmap. Colors from blue to red indicate the DEL expression level from low to high. Median risk score is represented by the dotted line, by which patients with BLCA were separated into the low- and high-risk score groups.

perform a multivariate Cox proportional hazards regression analysis (Table 1). A total of four DELs with $\mathrm{P}<0.005$ were chosen to construct a prognostic signature, including AC145124.1, AC010168.2, MIR200CHG, and AC098613.1 (Table 2, Fig. 4). Subsequently, the risk score used to predict OS was calculated using the following formula: Risk score $=\operatorname{EXP}_{\mathrm{AC} 145124.1}{ }^{*}(-0.105)+$ $\mathrm{EXP}_{\mathrm{AC} 010168.2} *(-0.427)+\mathrm{EXP}_{\mathrm{MIR} 200 \mathrm{CHG}} *(-0.181)+\operatorname{EXP}_{\mathrm{AC} 098613.1} *(-0.173)$.

BLCA patients were classified into two low- and high-risk subgroups based on the median level of the prognostic risk score (Fig. 5). The survival condition of BLCA patients could reliably be predicted by the risk score, with an AUC value of 0.734 (Fig. 6A). Furthermore, 
Fig. 6. Prognostic value of the risk score constructed from four DELs in BLCA. (A) Prognostic value of the risk score displayed as a timedependent ROC curve for predicting the survival status. (B) Kaplan-Meier analysis of the risk score for overall survival. Note: AUC, area under curve; CI, confidence interval.

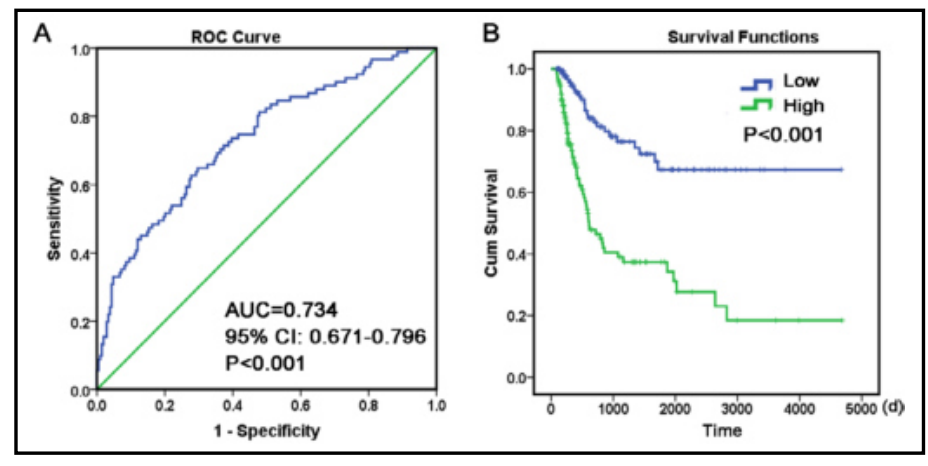

Table 3. Predictive values of related clinical parameters and risk score. Note: BMI, body mass index; CRR, Complete remission/ response; $\mathrm{SD}$, stable disease; $\mathrm{PRR}$, partial remission/response; $\mathrm{PD}$, progressive disease; HR, hazard ratio

\begin{tabular}{|c|c|c|c|c|c|}
\hline \multirow{3}{*}{$\begin{array}{l}\text { Variables } \\
\text { Gender }\end{array}$} & \multirow{2}{*}{$\begin{array}{c}\text { Patients } \\
\mathrm{n}=317\end{array}$} & \multicolumn{2}{|c|}{ Univariate analysis } & \multicolumn{2}{|c|}{ Multivariate analysis } \\
\hline & & $\mathrm{HR}(95 \% \mathrm{Cl})$ & Pvalue & $\mathrm{HR}(95 \% \mathrm{Cl})$ & $P$ value \\
\hline & & & & & \\
\hline male & 231 & 1.000 (Reference) & & & \\
\hline female & 86 & $1.199(0.778-1.847)$ & 0.41 & & \\
\hline BMI & 274 & & & & \\
\hline$<25$ & 95 & 1.000 (Reference) & & & \\
\hline$\geqslant 25$ & 179 & $0.802(0.507-1.268)$ & 0.346 & & \\
\hline Person Neoplasm Cancer Status & 286 & & & & \\
\hline tumor free & 165 & 1.000 (Reference) & & & \\
\hline with tumor & 121 & $9.803(5.392-17.822)$ & $<0.001$ & $7.067(2.057-24.279)$ & 0.002 \\
\hline Tobacco smoking history & 307 & & & & \\
\hline$<2.46$ & 134 & 1.000 (Reference) & & & \\
\hline$\geqslant 2.46$ & 173 & $1.241(0.82-1.878)$ & 0.307 & & \\
\hline Primary therapy outcome & 190 & & & & \\
\hline $\mathrm{CRR}+\mathrm{SD}+\mathrm{PRR}$ & 151 & 1.000 (Reference) & & & \\
\hline PD & 39 & $7.711(4.323-13.757)$ & $<0.001$ & $2.814(1.274-6.217)$ & 0.011 \\
\hline Diagnosis subtype & 313 & & & & \\
\hline non-papillary & 225 & 1.000 (Reference) & & & \\
\hline papillary & 88 & $0.589(0.348-0.996)$ & 0.048 & $1.695(0.687-4.180)$ & 0.252 \\
\hline Pathologic T stage & 288 & & & & \\
\hline T0-T2 & 84 & 1.000 (Reference) & & & \\
\hline T3-T4 & 204 & 2.777 (1.594-4.838) & $<0.001$ & $0.991(0.259-3.797)$ & 0.989 \\
\hline Lymphovascular invasion & & & & & \\
\hline No & 103 & 1.000( & & & \\
\hline Yes & 114 & $2.767(1.620-4.725)$ & $<0.001$ & $1.758(0.632-4.889)$ & 0.280 \\
\hline Pathologic N stage & 287 & & & & \\
\hline No & 178 & 1.000 (Reference) & & & \\
\hline N1-N3 & 109 & $2.54(1.654-3.902)$ & $<0.001$ & $0.486(0.182-1.297)$ & 0.149 \\
\hline Pathologic M stage & 146 & & & & \\
\hline a & 137 & 1.000 (Reference) & & & \\
\hline M1 & 9 & $3.271(0.979-10.933)$ & 0.054 & & \\
\hline Pathologic stage & 315 & & & & \\
\hline Stage I-II & 90 & 1.000 (Reference) & & & \\
\hline Stage III-IV & 225 & $3.765(2.008-7.059)$ & $<0.001$ & $4.836(0.794-29.454)$ & 0.087 \\
\hline New tumor event after initial treatment & 231 & & & & \\
\hline No & 163 & 1.000 (Reference) & & & \\
\hline Yes & 68 & $3.16(1.897-5.263)$ & $<0.001$ & $0.692(0.292-1.640)$ & 0.403 \\
\hline Clinical T stage & 139 & & & & \\
\hline T1-T2 & 105 & 1.000 (Reference) & & & \\
\hline T3-T4 & 34 & $3.146(1.606-6.159)$ & 0.001 & & \\
\hline Risk score & 302 & & & & \\
\hline Low & 159 & 1.000 (Reference) & & & \\
\hline High & 141 & $3.503(2.229-5.504)$ & $<0.001$ & $3.723(1.589-8.727)$ & 0.002 \\
\hline
\end{tabular}

Kaplan-Meier analysis indicated that the survival time of BLCA patients in the low-risk group was 3417.329 $\pm \quad 221.376$ days, which was predominantly more favorable than that of the high-risk group (1580.722 \pm 216.841 days, $\mathrm{P}<0.001$, Fig. 6B).

Meanwhile, we also evaluated the prognosticvalue of the clinical parameters of BLCA patients by univariate Cox proportional hazards regression analysis. A number of parameters could be used to predict the survival of BLCA patients (Table 3). However, after multivariate Cox proportional hazards regression analysis, only person neoplasm cancer status, primary therapy outcome success, pathologic stage and risk score were independent prognostic indictors of BLCA (Table 3). The Kaplan-Meier curves of these survival-associated clinical parameters are displayed in Fig. 7. The expression levels of the four DELs used to construct the prognostic signature in the low- and high-risk groups are shown in Fig. 8.

\section{Verification of the four-DELs risk scores using other databases}

Two microarrays were obtained from GEO databases based on the inclusion criteria, GSE89006 and GSE51493. However, only the expression data of AC010168.2 and AC098613.1 could be obtained (Fig. 9). In the GSE89006 microarray, AC010168.2 expression level in tumor tissue was slightly higher than that in normal tissue (Fig. 9A), which was consistent with that in TCGA (Fig. 4C). The expression trend of AC145124.1 in GSE89006 was contradictory with that in TCGA (data not shown). In GSE51493, the expression of AC098613.1 in tumor tissue was slightly higher than that in non-tumor tissue (Fig. 9B), which was consistent with that in TCGA (Fig. 4G). However, the expression trend of AC010168.2 in GSE51493 was inconsistent with that in TCGA (data not shown). 


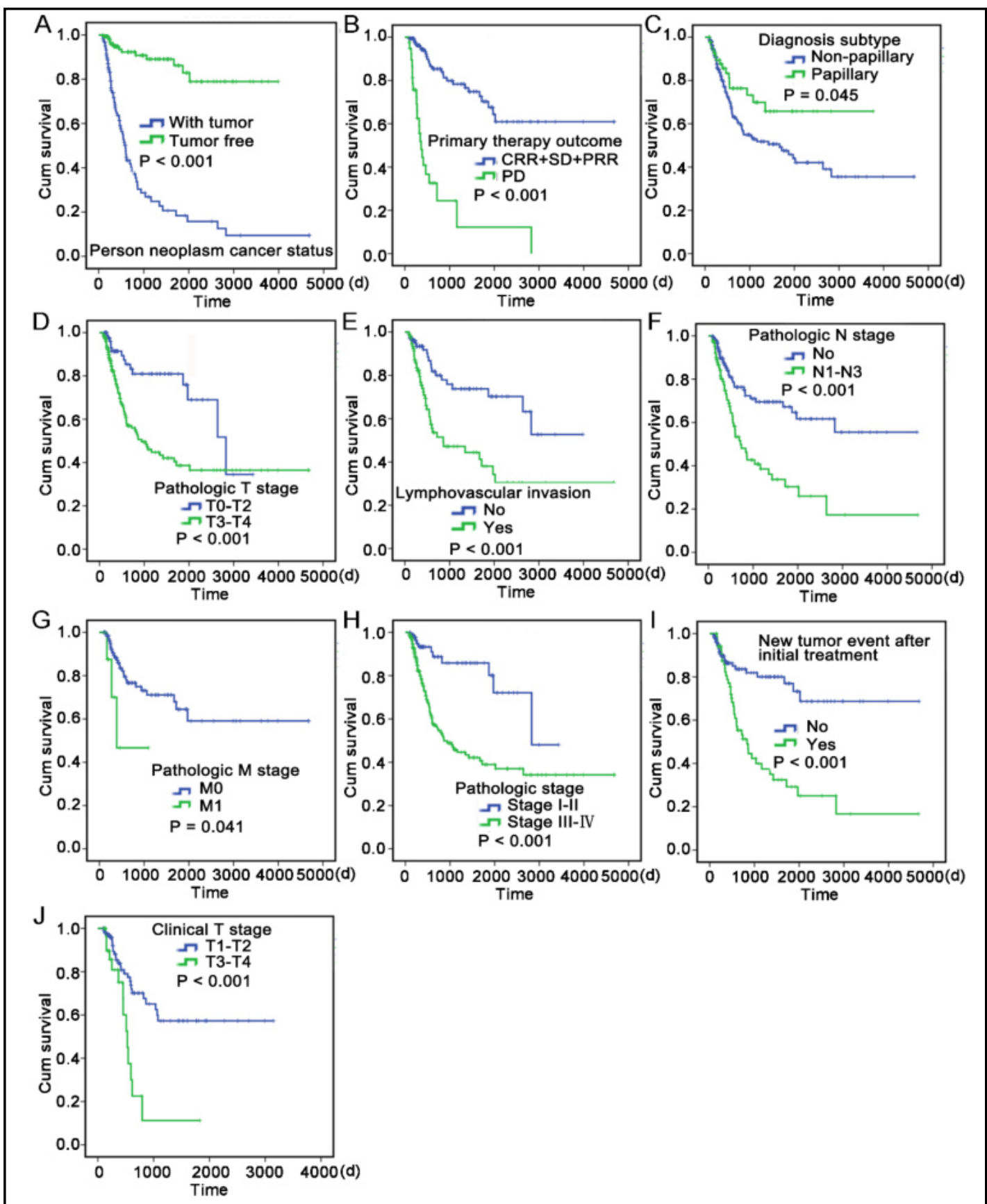

Fig. 7. Prognostic significance of 10 clinical parameters for the survival of BLCA patients. Kaplan-Meier analyses of 10 clinical parameters: (A) person neoplasm cancer status; (B) successful outcome of primary therapy; (C) diagnosis subtype; (D) pathologic T stage; (E) lymphovascular invasion; (F) pathologic $N$ stage; (G) pathologic M stage; (H) pathologic stage; (I) new tumor event; and (J) clinical T stage.

The expression data of only AC010168.2 could be extracted from both GSE51493 and GSE89006. Subsequently, a meta-analysis of AC010168.2 was performed with data from TCGA, GSE51493, and GSE89006 based on 26 non-cancerous controls and 430 BLCA patients. The results showed that the SMD was $0.77(-0.99,2.53)$ (Fig. 10). Furthermore, heterogeneity tests (I2) showed that $\mathrm{P}=0.002$ with $\mathrm{I} 2$ of $83.5 \%$ (Fig. 10A) and one study is outside $95 \% \mathrm{CI}$ in the Begg's funnel plot (Fig. 10B). The source of the heterogeneity may come from the different tissue of control group in the three studies. The control group of GSE51493 is the 
Fig. 8. Comparison of the expression of four DELs between the low- and high-risk score groups. Expression levels of (A) AC145124.1, (B) AC010168.2, (C) MIR200CHG and (D) AC098613.1 between the low- and high-risk score groups.

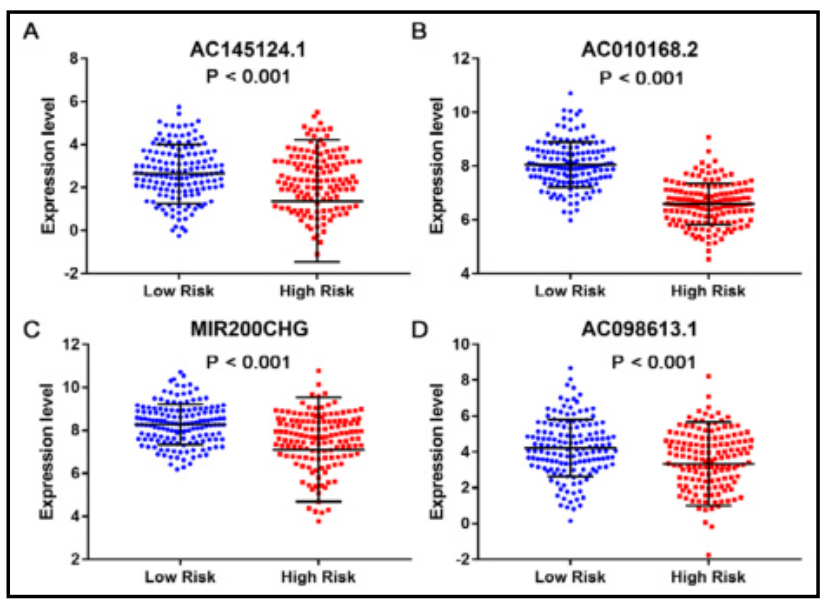

Fig. 9. Expression levels of the prognostic DELs from GEO datasets. (A) GSE89006, and (B) GSE51493.

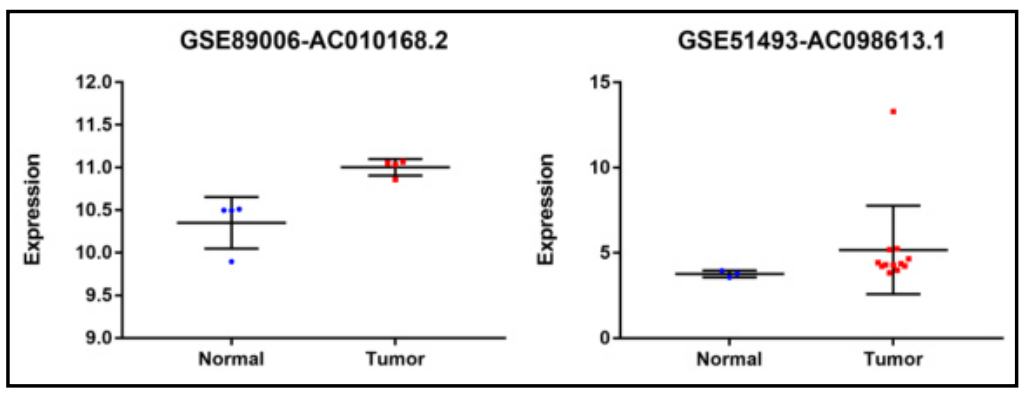

Fig. 10. Expression level of AC010168.2 by meta-analysis. (A) Forest plot; and (B) publication bias. Standard mean difference (SMD) was calculated based on 430 BLCA cases and 26 non-cancerous controls.

normal bladder mucosa tissue while the control groups of TCGA and GSE89006 are both para-carcinoma tissue. This suggests that AC010168.2 expression may differ between normal bladder and paracarcinoma tissue.

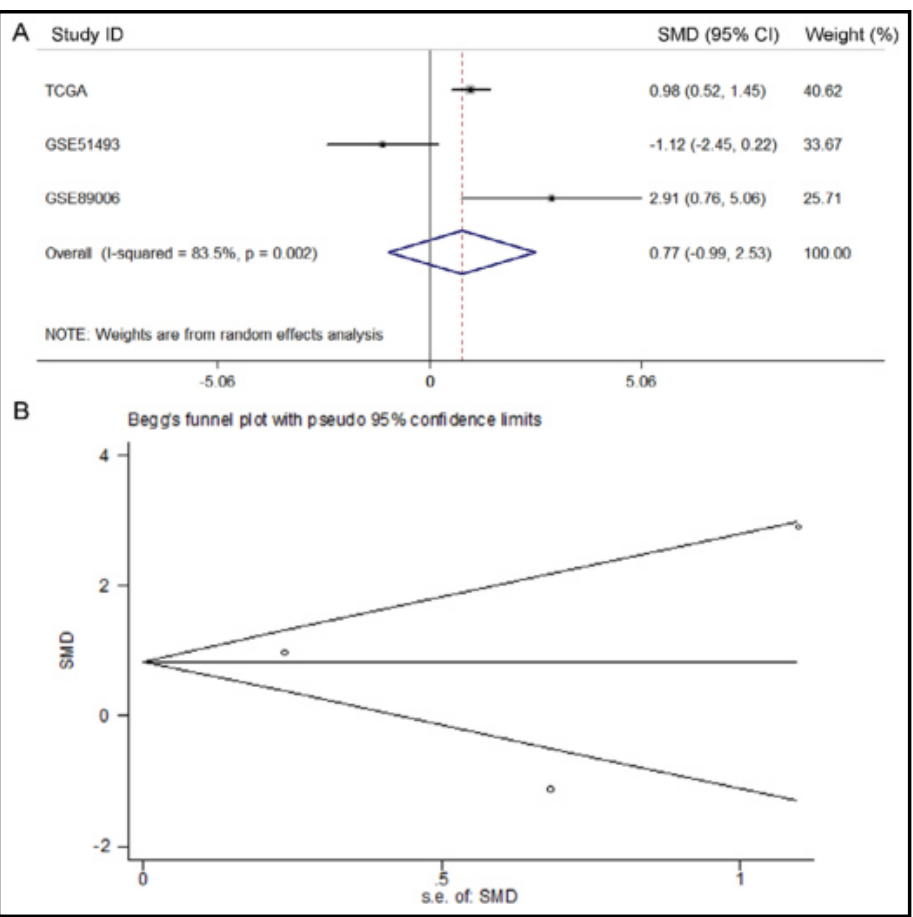

The expression data of these four lncRNAs in 31 types of tumors from GEPIA indicated that AC145124.1 and AC010168.2 are expressed in almost all tumor types; however, MIR200CHG and AC098613.1 showed no expression in several tumors, such as adrenocortical carcinoma and uterine carcinosarcoma (Fig. 11). 
Fig. 11. Expression $A$ levels of four IncRNAs in 31 cancers from GEPIA data.

(A)AC145124.1, (B) AC010168.2, MIR200CHG, and (D) AC098613.1. Note: ACC, Adrenocortical carcinoma; BLCA, Bladder urothelial carcinoma; BRCA, Breast invasive carcinoma; CESC, Cervical squamous cell carcinoma and endocervical adenocarcinoma; CHOL, Cholangiocarcinoma; COAD, Colon adenocarcinoma; DLBC, Lymphoid Neoplasm Diffuse Large B-cell Lymphoma; ESCA, Esophageal carcinoma; GBM, Glioblastoma multiforme; HNSC, Head and Neck squamous cell carcinoma; $\mathrm{KICH}$, Kidney Chromophobe; KIRC, Kidney renal clear cell carcinoma; KIRP,

Kidney renal papillary cell carcinoma; LAML, Acute Myeloid Leukemia; LGG, Brain Lower Grade

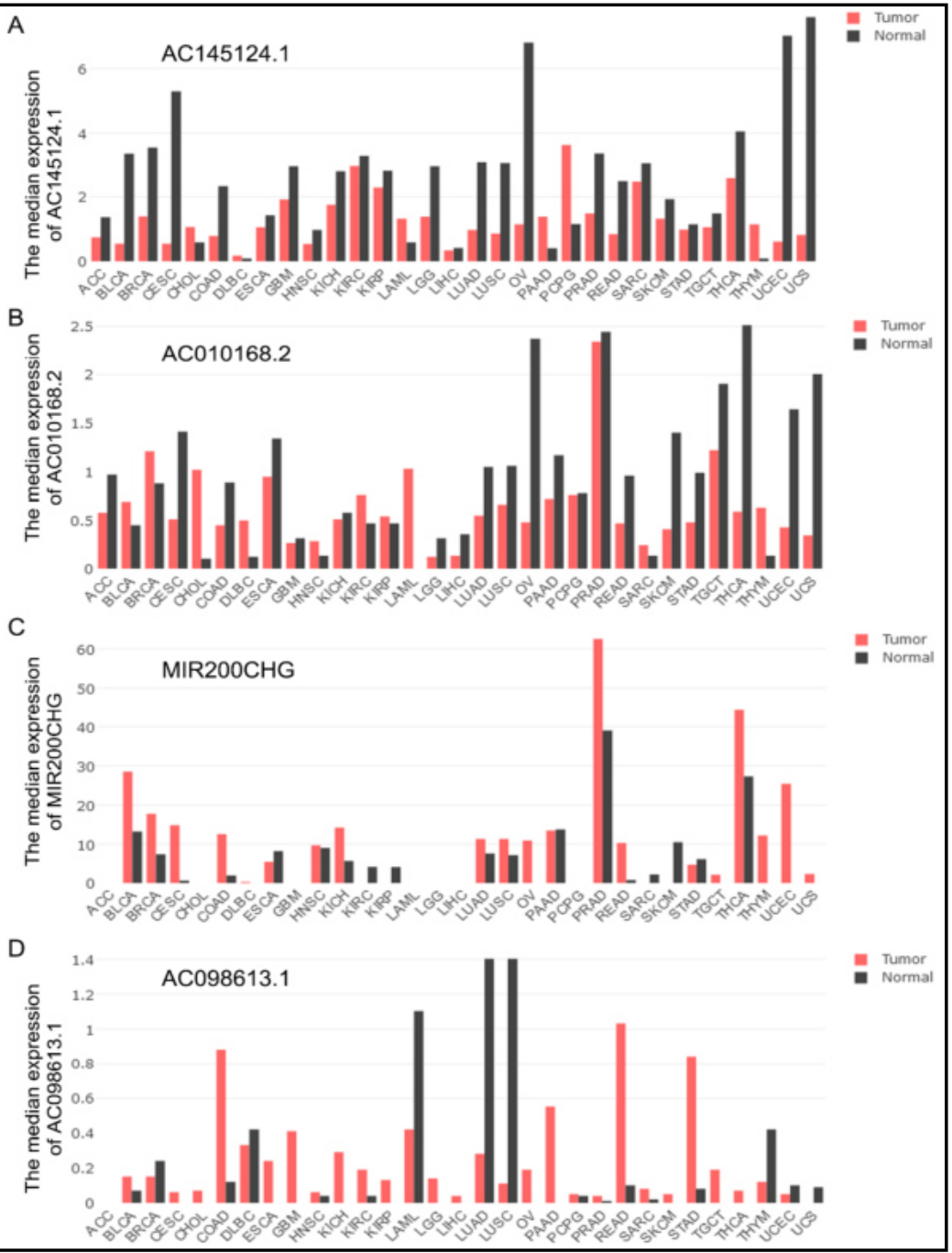
Glioma; LIHC, Liver hepatocellular carcinoma; LUAD, Lung adenocarcinoma; LUSC, Lung squamous cell carcinoma; MESO, Mesothelioma; OV, Ovarian serous cystadenocarcinoma; PAAD, Pancreatic adenocarcinoma; PCPG, Pheochromocytoma and Paraganglioma; PRAD, Prostate adenocarcinoma; READ, Rectum adenocarcinoma; SARC, Sarcoma; SKCM, Skin cutaneous Melanoma; STAD, Stomach adenocarcinoma; TGCT, Testicular Germ Cell Tumors; THCA, Thyroid carcinoma; THYM, Thymoma; UCEC, Uterine Corpus Endometrial Carcinoma; UCS, Uterine Carcinosarcoma.

The expression of the four IncRNAs in cell lines and BLCA tissue samples assessed in-house

RT-qPCR results showed that the expression of AC145124.1, AC010168.2, MIR200CHG, and AC098613.1 was detected in five cell lines, but at different levels (Fig. 12A). Since there was only one non-cancerous control cell line, no statistical analysis was possible. Moreover, the expression of the four IncRNAs could also be detected in BLCA tissue samples (Fig. 12B). Unfortunately, no adjacent tumor tissue could be analyzed due to the specificity of operation strategy.

Network of genes co-expressed with the prognostic DELS

To evaluate the possible function and molecular mechanism of the four prognostic DELs, co-expressed genes were identified from the MEM database (http://biit.cs.ut.ee/mem/). However, data for only the co-expressed genes AC145124.1, AC010168.2, and MIR200CHG could be downloaded. The co-expressed genes AC145124.1, with a score less than 10E-15, 
AC010168.2 and MIR200CHG, with scores less than 10E-30, were selected for gene network analysis, as shown in Figures 13, 14, and 15.

Fig. 12. Expression of the four lncRNAs in five BLCA cell lines and tissue samples.

Expression of the four IncRNAs in BLCA cell lines SW780, 5637, J82, and T24 and the human uroepithelial cell

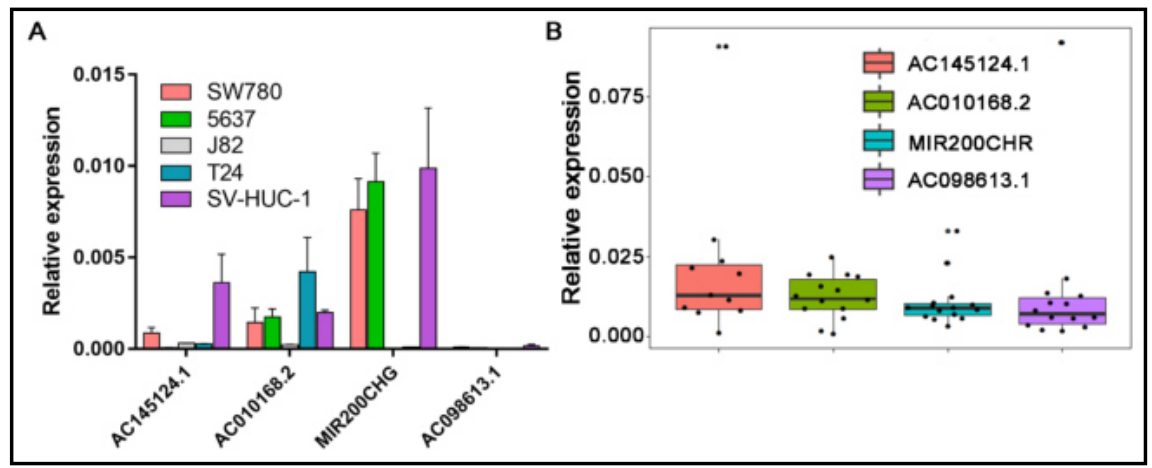
SV-HUC-1 cell line. (B) Expression of the four lncRNAs in 15 BLCA tissue samples collected from the First Affiliated Hospital of Guangxi Medical University.

Fig. 13. Gene network of the co-expressed genes of AC145124.1. (A) Network of genes co-expressed with AC145124.1. DELs are represented by dark blue triangles and mRNAs by light blue rectangles. (B) protein-protein interaction (PPI) network of coexpressed genes. Genes not connected to the network are not shown.

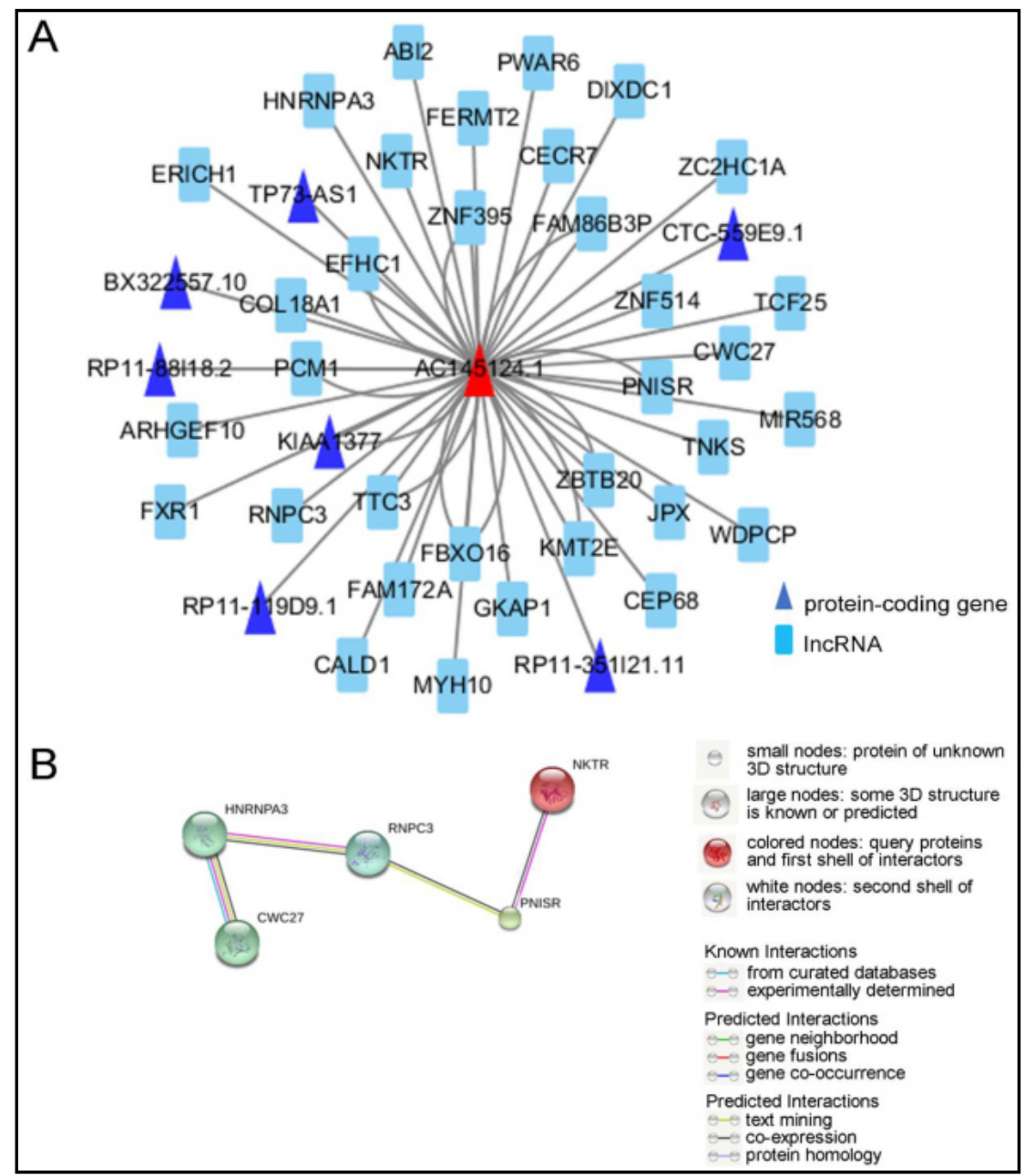


Fig. 14. Gene network of the coexpressed genes of AC010168.2. Network of genes co-expressed with AC010168.2. DELS are represented by dark blue triangles and mRNAs by light blue rectangles. (B) PPI network of coexpressed genes. Genes not connected to the network are hidden.

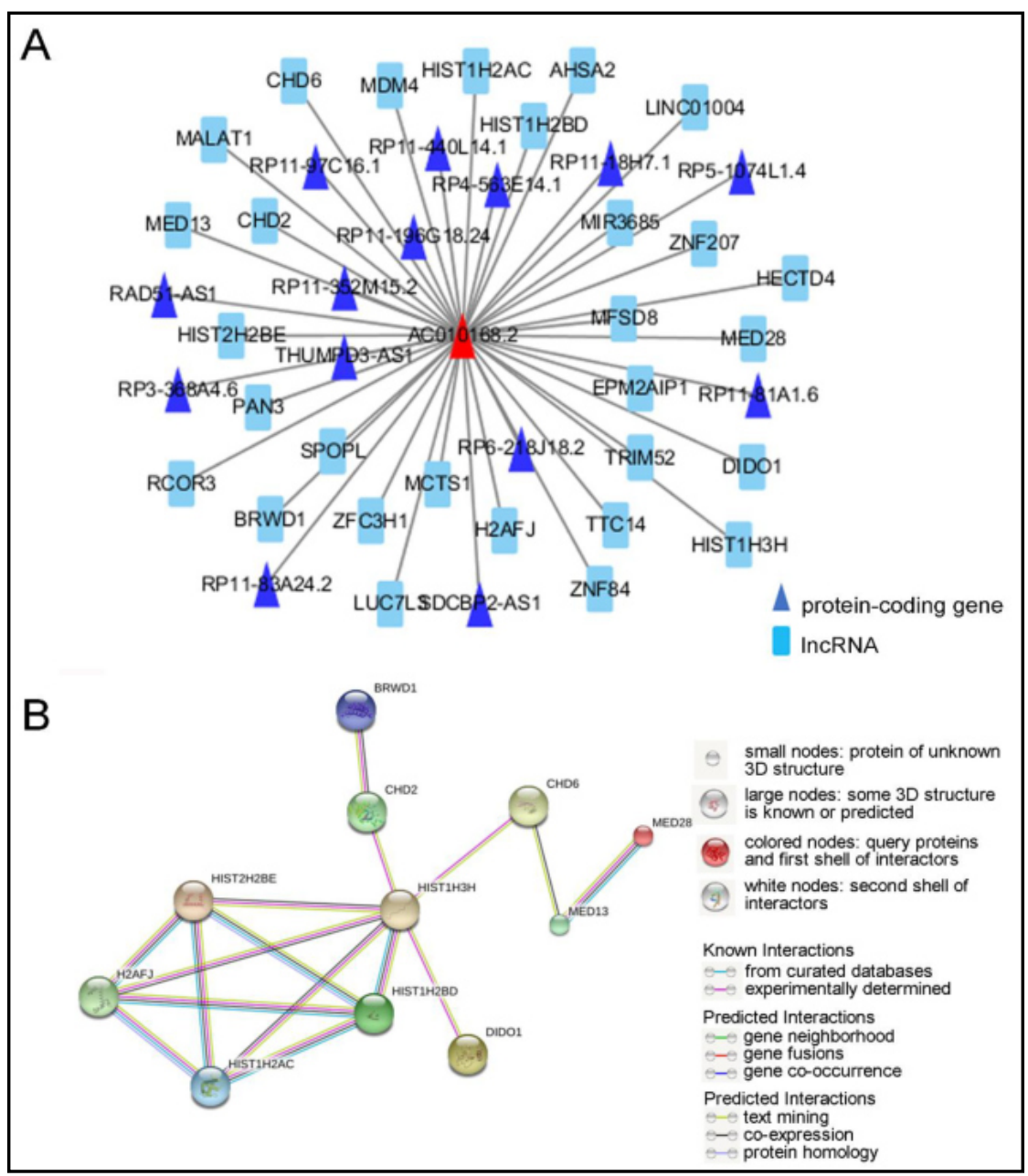

Acquisition and functional assessment of DEGs in BLCA between low-and high-risk score groups

DEGs from both the low- and high-risk groups were obtained using the R package edgeR as described above, provided by the BLCA RNA-seq data downloaded from TCGA. DEGs in the low-risk group comprised 1475 genes with $\mathrm{P}<1 \mathrm{E}-10$, and there were 975 genes with $\mathrm{P}<1 \mathrm{E}$ 10 in the high-risk group. The DEGs were then used for GO and Kyoto Encyclopedia of Genes and Genomes (KEGG) pathway analyses. The top five GO and KEGG pathways are shown in Tables 4 and 5 and Figures 16 and 17. There were four common pathways among the top five KEGG pathways, as follows: 'Dilated cardiomyopathy', 'cGMP-PKG signaling pathway', 'Oxytocin signaling pathway', and 'Calcium signaling pathway'. However, the 'Vascular smooth muscle contraction' pathway appeared in the low-risk group while the 'Hypertrophic cardiomyopathy' pathway was identified in the high-risk group.

Disease ontology (DO) analysis showed that DEGs from the high-risk groups were significantly enrichedinmusculardisease, musculartissuedisease, myopathy, cardiomyopathy, intrinsic cardiomyopathy, muscular dystrophy, hypertrophic cardiomyopathy, and Duchenne muscular dystrophy (Fig. 18), while the DEGs from the low-risk groups were remarkably enriched in tetralogy of Fallot, glycogen storage disease, and disorders of glycogen metabolism (Fig. 19).

Potential biological function of the prognostic DEL AC010168.2

We further investigated if there were any protein-coding genes adjacent to the DELs. Interestingly, the protein-coding gene HIST4H4 was found $68 \mathrm{bp}$ upstream of the 
Fig. 15. Gene network of the coexpressed genes of MIR200CHG. Network of genes co-expressed with MIR200CHG. DELS are represented by dark blue triangles and mRNAs by light blue rectangles. (B) PPI network of coexpressed genes. Genes not connected to the network are hidden.

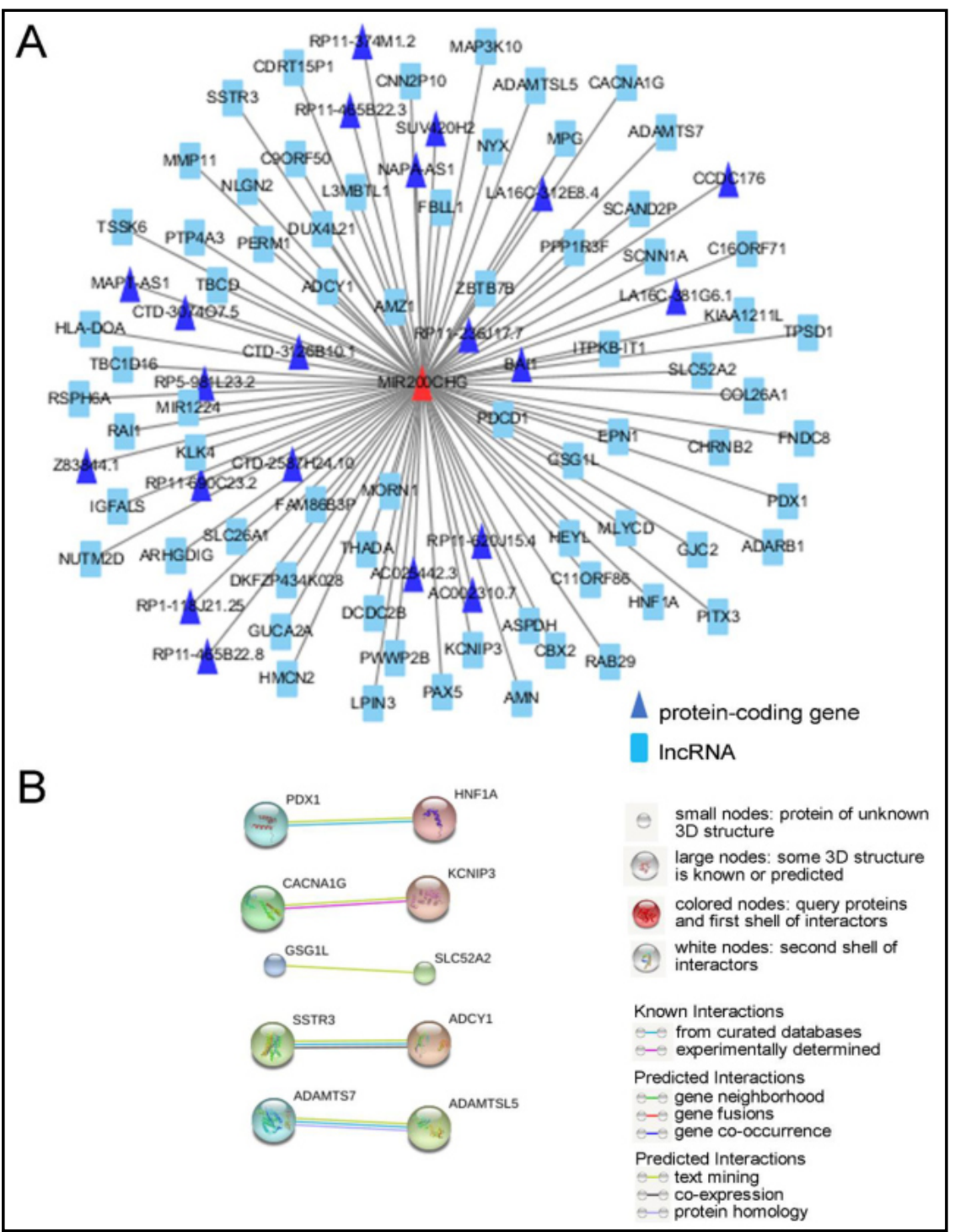

Table 4. GO and KEGG pathways of the DEGs in the low-risk group. Note: GO, gene ontology; KEGG, Kyoto Encyclopedia of Genes and Genomes; DEGs, differentially expressed genes; CC, cellular component; MF, molecular function; BP, biological process

\begin{tabular}{|c|c|c|c|}
\hline Term & Count & Total gene & P value \\
\hline GO BP: 0006936 muscle contraction & 34 & 1147 & $6.70 \mathrm{E}-14$ \\
\hline GO BP: 0007517 muscle organ development & 27 & 1147 & $1.17 \mathrm{E}-10$ \\
\hline GO BP: 0007155 cell adhesion & 68 & 1147 & $2.49 \mathrm{E}-09$ \\
\hline GO BP: 0009612 response to mechanical stimulus & 19 & 1147 & $4.00 \mathrm{E}-08$ \\
\hline GO BP: 0008016 regulation of heart contraction & 13 & 1147 & $4.03 \mathrm{E}-07$ \\
\hline GO CC: $0030018 \sim \mathrm{Z}$ disc & 45 & 1200 & $1.94 \mathrm{E}-22$ \\
\hline GO CC: 0042383 sarcolemma & 30 & 1200 & $4.79 \mathrm{E}-14$ \\
\hline GO CC: 0015629 actin cytoskeleton & 40 & 1200 & $9.57 \mathrm{E}-09$ \\
\hline GO CC: $0005886 \sim$ plasma membrane & 353 & 1200 & $1.18 \mathrm{E}-08$ \\
\hline GO CC: $0005925 \sim$ focal adhesion & 58 & 1200 & $1.18 \mathrm{E}-08$ \\
\hline GO MF: 0001077 transcriptional activator activity, RNA polymerase II core promoter proximal region sequence-specific binding & 43 & 1128 & $4.58 \mathrm{E}-09$ \\
\hline GO MF: 0008201 heparin binding & 33 & 1128 & $1.81 \mathrm{E}-08$ \\
\hline GO MF: 0008307 structural constituent of muscle & 16 & 1128 & $3.79 \mathrm{E}-08$ \\
\hline GO MF: 0005509 calcium ion binding & 81 & 1128 & 3.67E-06 \\
\hline GO MF: 0044325 ion channel binding & 23 & 1128 & $4.73 \mathrm{E}-06$ \\
\hline KEGG: hsa04022-cGMP-PKG signaling pathway & 46 & 491 & $8.25 \mathrm{E}-16$ \\
\hline KEGG: hsa04921-0xytocin signaling pathway & 39 & 491 & $9.66 \mathrm{E}-12$ \\
\hline KEGG: hsa04270-Vascular smooth muscle contraction & 33 & 491 & $1.89 \mathrm{E}-11$ \\
\hline KEGG: hsa04020-Calcium signaling pathway & 40 & 491 & $1.29 \mathrm{E}-10$ \\
\hline KEGG: hsa05414-Dilated cardiomyopathy & 25 & 491 & $1.75 \mathrm{E}-09$ \\
\hline
\end{tabular}


Table 5. GO and KEGG pathways of the DEGs in the high-risk group. Note: GO, gene ontology; KEGG, Kyoto Encyclopedia of Genes and Genomes; DEGs, differentially expressed genes; CC, cellular component; MF, molecular function; BP, biological process

\begin{tabular}{|c|c|c|c|}
\hline Term & Count & Total gene & P value \\
\hline GO BP: 0006936 muscle contraction & 33 & 786 & $8.72 \mathrm{E}-18$ \\
\hline GO BP: 0030198 extracellular matrix organization & 32 & 786 & $2.38 \mathrm{E}-09$ \\
\hline GO BP: 0007067 mitotic nuclear division & 36 & 786 & $5.00 \mathrm{E}-09$ \\
\hline GO BP: 0008016 regulation of heart contraction & 13 & 786 & $6.22 \mathrm{E}-09$ \\
\hline GO BP: 0051301 cell division & 44 & 786 & $6.78 \mathrm{E}-09$ \\
\hline GO CC: $0030018 \sim Z$ disc & 37 & 822 & $1.14 \mathrm{E}-20$ \\
\hline GO CC: 0042383 sarcolemma & 27 & 822 & $2.65 \mathrm{E}-15$ \\
\hline GO CC: $0005737 \sim$ cytoplasm & 304 & 822 & $1.04 \mathrm{E}-07$ \\
\hline GO CC: $0015629 \sim$ actin cytoskeleton & 29 & 822 & $5.92 \mathrm{E}-07$ \\
\hline GO CC: $0030017 \sim$ sarcomere & 12 & 822 & 7.63E-07 \\
\hline GO MF: 0005515 protein binding & 481 & 778 & $1.39 \mathrm{E}-08$ \\
\hline GO MF: 0008307 structural constituent of muscle & 14 & 778 & $2.78 \mathrm{E}-08$ \\
\hline GO MF: 0008201 heparin binding & 24 & 778 & $1.19 \mathrm{E}-06$ \\
\hline GO MF: 0001077 transcriptional activator activity, RNA polymerase II core promoter proximal region sequence-specific binding & 30 & 778 & $1.43 \mathrm{E}-06$ \\
\hline GO MF: 0003779 actin binding & 32 & 778 & $4.95 \mathrm{E}-06$ \\
\hline KEGG: hsa05410-Hypertrophic cardiomyopathy & 21 & 893 & 2.351624 \\
\hline KEGG: hsa05414-Dilated cardiomyopathy & 20 & 893 & 2.239642 \\
\hline KEGG: hsa04022-cGMP-PKG signaling pathway & 28 & 893 & 3.135498 \\
\hline KEGG: hsa04921-Oxytocin signaling pathway & 25 & 893 & 2.799552 \\
\hline KEGG: hsa04020-Calcium signaling pathway & 26 & 893 & 2.911534 \\
\hline
\end{tabular}

Fig. 16. GO and KEGG analyses of differentially expressed genes (DEGs) in the lowrisk group. The degree of enrichment is represented by the rich factor, which is the ratio between the number of selected genes for a term and the total number of genes in the analysis. The size of each node indicates the total number of genes

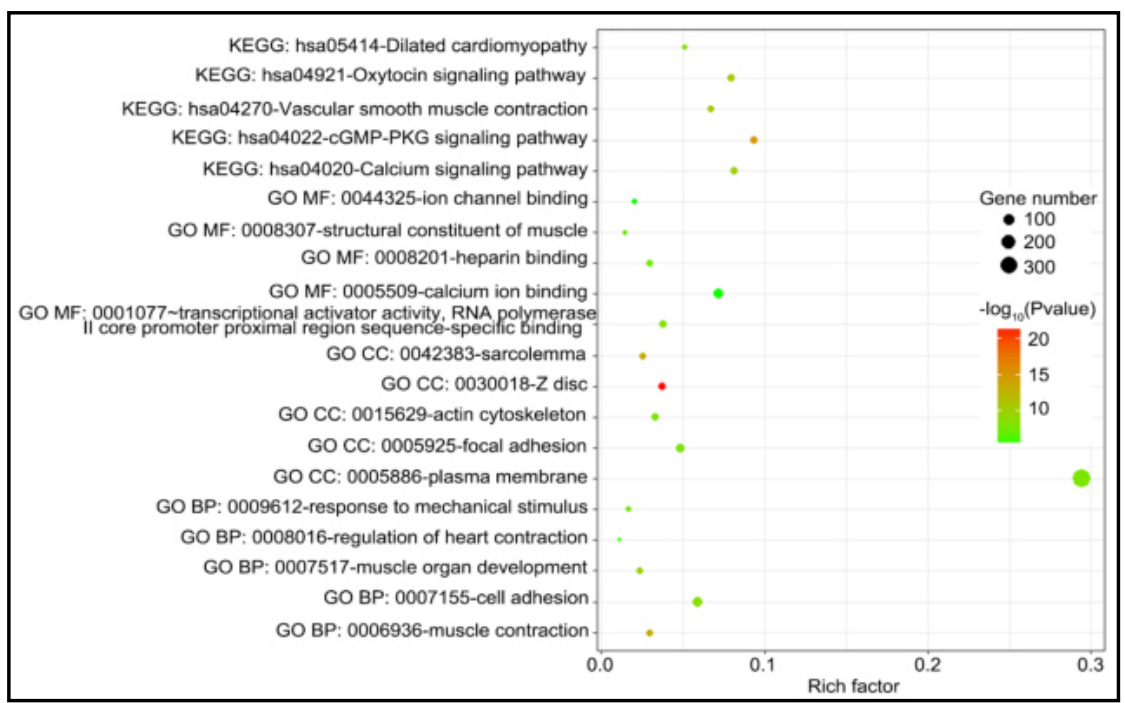
involved and the color reflects the P value from the enrichment analysis. Note: GO, gene ontology; KEGG, Kyoto Encyclopedia of Genes and Genomes; CC, cellular component; MF, molecular function; BP, biological process.

DEL AC010168.2 (Fig. 20A). HIST4H4 mRNA level was markedly higher in BLCA tissue $(5.713 \pm 1.227)$ than in non-cancerous bladder tissue $(4.891 \pm 0.926, \mathrm{P}=0.014$, Fig. $20 \mathrm{~B})$. The ROC curve revealed that HIST4H4 expression could distinguish BLCA from non-cancerous bladder tissue with an AUC of 0.716 based on TCGA data (Fig. 20C). Correlation analysis also revealed a positive relationship between AC010168.2 and HIST4H4 levels (Pearson's correlation coefficient $=0.824, \mathrm{P}<0.001$, Fig. 20D). In-house real time RT-qPCR showed that HIST4H4 mRNA was expressed at different levels in BLCA cell lines and tissue (Fig. $20 \mathrm{E})$. However, due to the absence of non-cancerous controls, no statistical analysis could be conducted to compare the expression difference of HIST4H4 mRNA between BLCA and non-cancerous bladder tissue. We also failed to perform prognostic analysis, due to the short time of follow-up. Excitingly, correlation analysis confirmed that the expression of AC010168.2 was significantly positively associated with that of HIST4H4 in BLCA tissue, as assessed by real time RT-qPCR (Fig. 20F). In addition, the protein expression assessed by immunohistochemistry from THPA database also suggested that HIST4H4 protein tended to be over-expressed in BLCA tissue (Fig. 21, Fig. 22) shown with several primary antibodies.

However, no other genes adjacent to AC010168.2 were significantly correlated with it 
Fig. 17. GO and KEGG analyses of the differentially expressed genes (DEGs) in the high-risk group. The degree of enrichment is represented by the rich factor, which is the ratio between the number of selected genes for a term and the total number of genes in the analysis. The size of each node indicates the total number of genes involved and the color reflects the P value from the enrichment analysis. Note: GO, gene ontology; KEGG, Kyoto Encyclopedia of Genes and Genomes; CC, cellular component; MF, molecular function; BP, biological process.

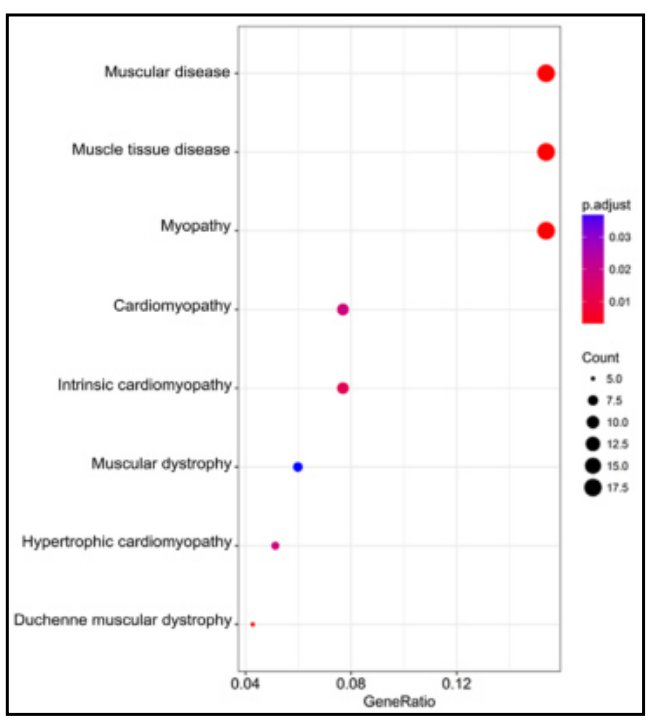

Fig. 18. Disease ontology (DO) analysis of the differentially expressed genes (DEGs) from the high-risk groups. $\mathrm{P}$ values represent the degree of enrichment. The count is the number of genes involved in the disease. Gene Ratio is the ratio between the number of selected genes for a disease and the total number of genes in the DO analysis.

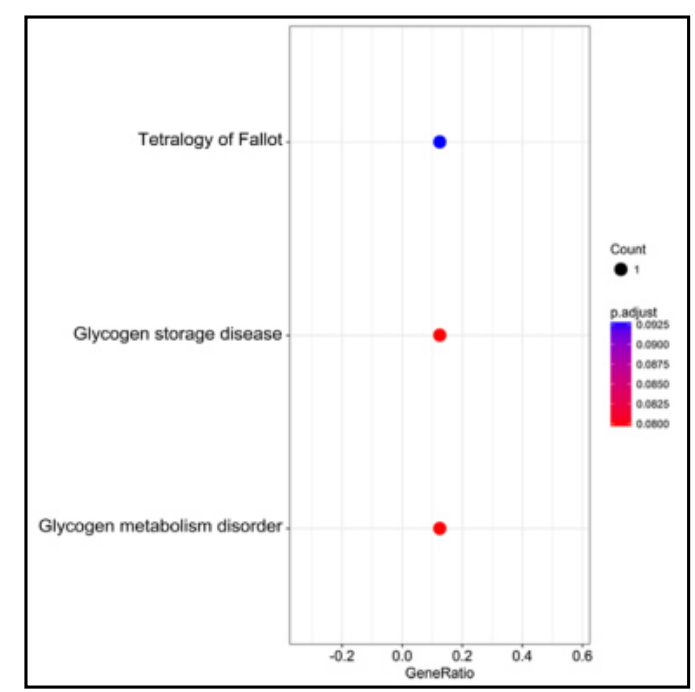

Fig. 19. Disease ontology analysis of the differentially expressed genes (DEGs) from the low-risk groups. $P$ values represent the degree of enrichment. The count is the number of genes involved in the disease. Gene Ratio is the ratio between the number of selected genes for a disease and the total number of genes in the DO analysis.

(data not shown). Meanwhile, no obvious correlations were observed between the other 3 DELs (AC145124.1, MIR200CHG OR AC098613.1) and their respective adjacent proteincoding genes (Supplementary data - for all supplemental material see www.karger. com/10.1159/000494647/). 


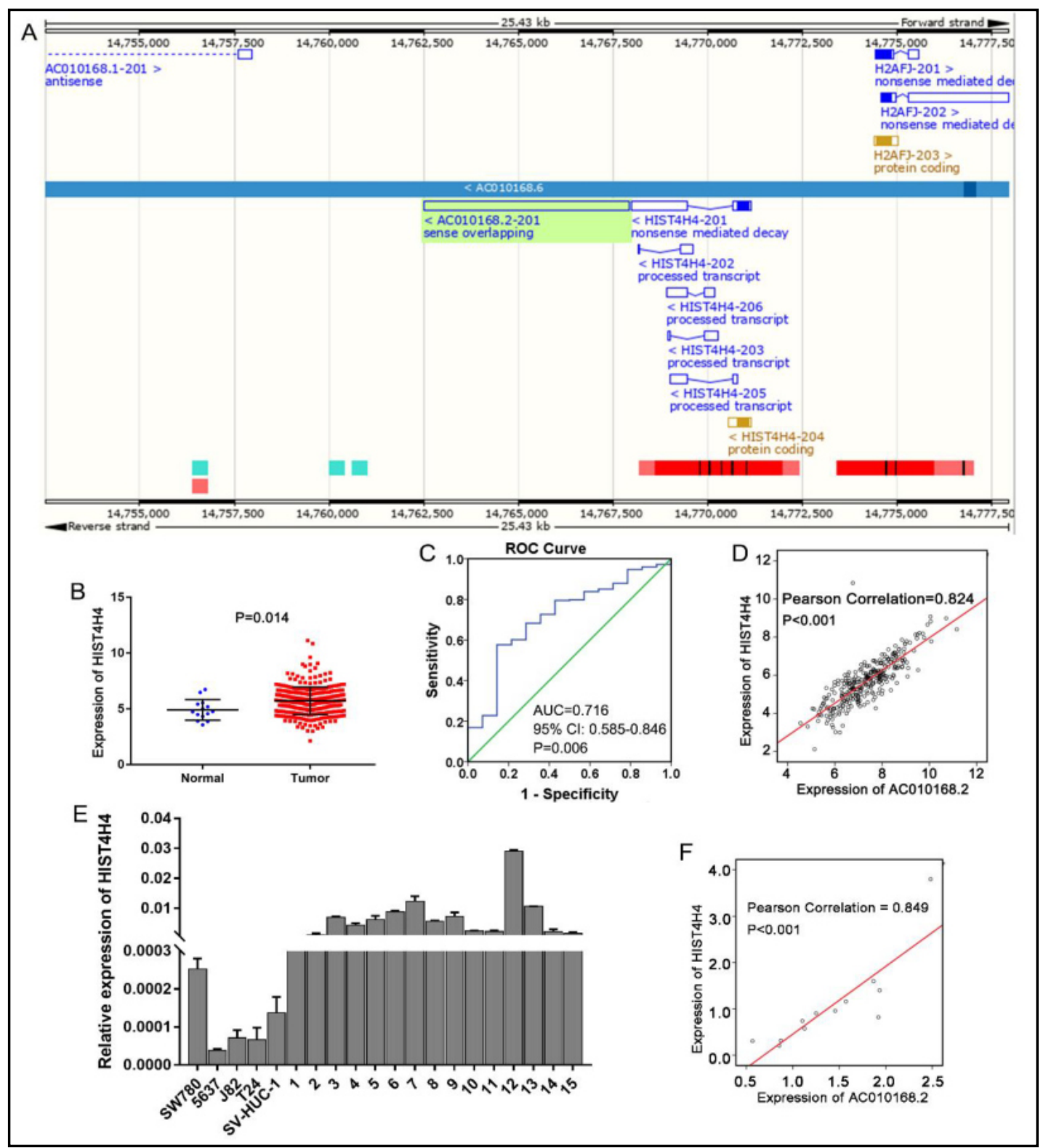

Fig. 20. Biological features of prognostic differentially expressed lncRNA (DEL) AC010168.2 and its influence on the adjacent protein-coding gene. (A) Relative genomic location of AC010168.2 and HIST4H4. LncRNA AC010168.2: Chromosome 12, 14,762,504-14,767,931 reverse strand. HIST4H4: Chromosome 12, 14,767,999-14,771,131 reverse strand. HIST4H4 is 68bp from AC010168.2. (B) Expression of HIST4H4 in non-tumor bladder and BLCA tissue from TCGA dataset. (C) ROC curve of HIST4H4 for predicting BLCA from TCGA data. (D) Pearson's correlation analysis of AC010168.2 and HIST4H4 in 317 tissue samples from TCGA. (E) Expression of HIST4H4 in five cell lines and 15 BLCA tissue samples from the First Affiliated Hospital of Guangxi Medical University. (F) Pearson's correlation analysis of the expression of AC010168.2 and HIST4H4 in 15 BLCA tissue samples. Note: HIST4H4, histone cluster 4 H4; AUC, area under the curve. 
Fig. 21. Protein expression of HIST4H4 as detected by the primary antibody HPA042201. Protein expression of HIST4H4 was provided by The Human Protein Atlas. (A) Normal bladder tissue. (B) Urothelial carcinoma tissue from female patient (ID 3079). (C) Urothelial carcinoma tissue from female patient (ID 3516). (D) Urothelial carcinoma tissue from female patient (ID 3079). Note: HIST4H4, histone cluster $4 \mathrm{H} 4$.

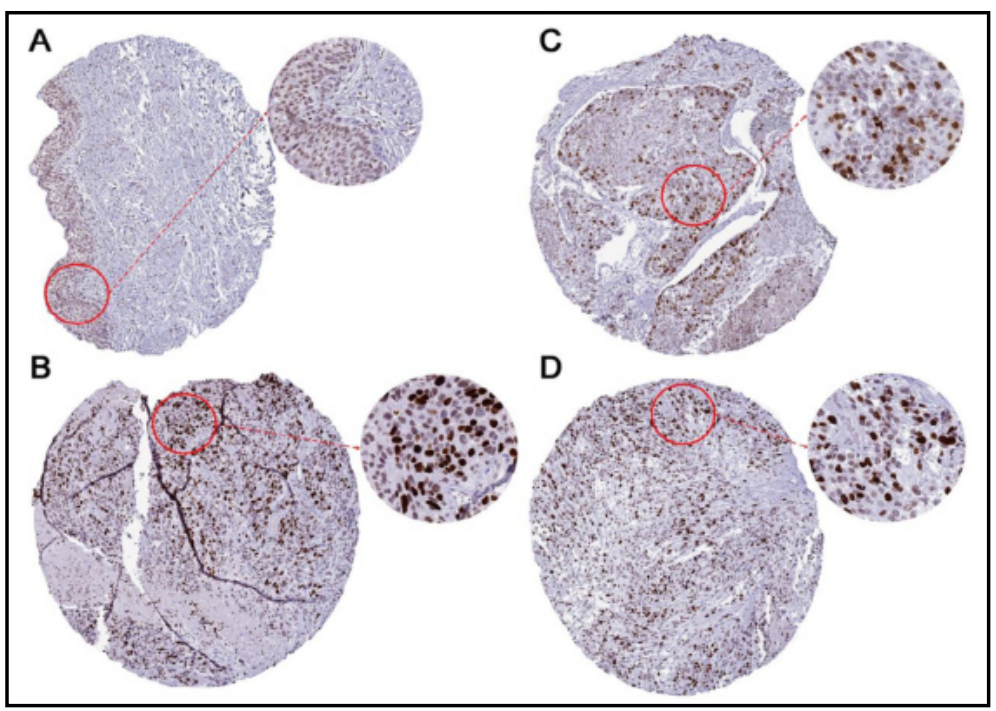

Fig. 22. Protein expression of HIST4H4 as detected by the primary antibody CAB011503. Protein expression of HIST4H4 was provided by The Human Protein Atlas. (A) Normal bladder tissue. (B) Urothelial carcinoma tissue from female patient (ID 3077). (C) Urothelial carcinoma tissue from female patient (ID 3077). (D) Urothelial carcinoma tissue from female patient (ID 3002). Note: HIST4H4, histone cluster $4 \mathrm{H} 4$.

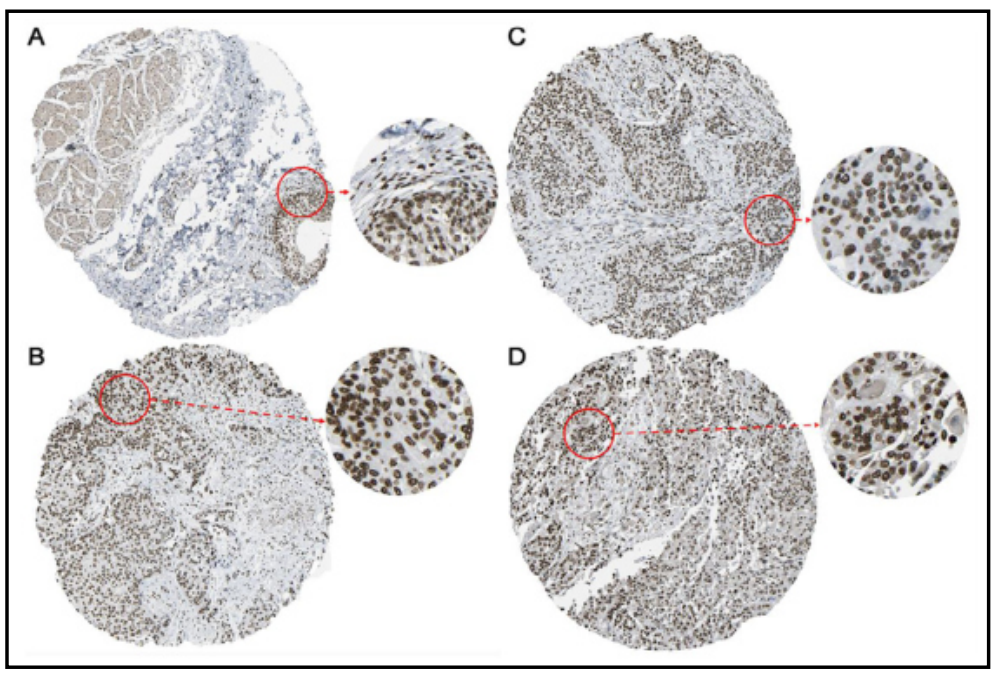

\section{Discussion}

First, due to the contributions of TCGA RNA-sequencing data, DELs in BLCA were identified in BLCA in this study, which was followed by univariate and multivariate Cox regression analyses. It is noteworthy that the risk score based on four novel DELs (AC145124.1, AC010168.2, MIR200CHG, and AC098613.1) could accurately predict the prognosis of BLCA patients. Furthermore, we found that distinct signaling pathways may exert their functions in the low- and high-risk groups. Lastly, a protein-coding gene, HIST4H4 was found just 68 $\mathrm{bp}$ from one of the DELs, AC010168.2. The expression of HIST4H4 was positively correlated with that of AC010168.2 in BLCA patients. This in silico investigation thereby pioneers the future investigation of prognostic lncRNAs for BLCA.

Prognostic IncRNAs have been previously reported by Bao et al. [24], who found that four lncRNAs, AC005682.5, CTD-2231H16.1, CTB-92J24.2, and RP11-727F15.13 could form a signature to predict survival in BLCA. Because the amount of data on BLCA patients in both TCGA and lncRNA datasets has been greatly expanded, we were able to perform this innovative investigation. Surprisingly, all four IncRNA candidates in our current study were different from those found by Bao et al. [24]. Compared to the previous study by Bao et al. 


\section{Cellular Physiology Cell Physiol Biochem 2018;50:1474-1495

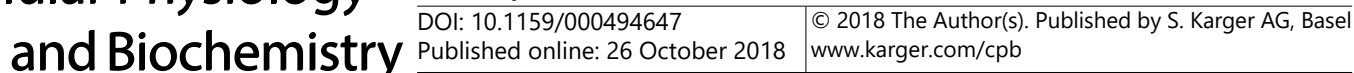

He et al.: a Prognostic Four-IncRNA-Based Risk Score For BLCA

[24], there are several advantages of the present study which need to be highlighted. First, Bao et al. [24] identified prognostic IncRNAs in a training group with 117 cases, then validated those lncRNAs in another subset of the cases as a testing group ( $\mathrm{n}=117)$ as well as with the entire group $(n=234)$. The data were downloaded in May of 2016. The present study covers a total of 414 BLCA patients, and 317 cases had sufficient data to identify novel prognostic lncRNAs, which thereby enlarges the sample size of inquiry. Second, our prognostic lncRNAs were discovered from 317 cases, a much larger cohort than that of Bao et al. [24], which provides more convincing results. Third, cohorts with alternative detection approaches (i.e., microarray) were used for validation in the current study, which further strengthens the current findings, as Bao et al. [24] had only a single cohort from TCGA. Even though we failed to perform meta-analyses for all four of these lncRNAs, in-house RT-qPCR could detect the expression of the lncRNAs both in BLCA cell lines and clinical samples. Fourth, the number of identified lncRNAs increased from 12730 to 13199 in the present study; 469 more new lncRNAs were found. More importantly, updated lncRNA annotations may have changed the known attributes of genes. One typical example is the IncRNA CTD-2231H16.1 from the study of Bao et al. [24], which has been excluded in the newly updated GENCODE. Fifth, before the prognostic evaluation, we screened out DELs that were inconsistent with the findings of Bao et al. [24]. If lncRNAs exerted little effect on carcinogenesis, their prognostic significance would be decreased. We investigated the prognostic value of the four DELs based on the evidence that they were differentially expressed between BLCA and non-cancerous bladder tissue. Accordingly, the four DELs (AC145124.1, AC010168.2, MIR200CHG, and AC098613.1) in the current study served as indicators of not only the stage of tumorigenesis, but also the process of deterioration. Sixth, Bao et al. [24] did not exclude patients with short survival times. In contrast, we only included patients whose survival time was longer than 90 days, as those with shorter survival times probably died of causes other than the cancer itself. Seventh, we attempted to explain the underlying molecular mechanism of these lncRNAs by performing signaling pathway analyses to compare the low- and high-risk groups. Lastly, adjacently located protein-coding genes were identified with the Ensemble Genome Browser. Thus, compared to the previous report, the current study yields more powerful and comprehensive data, showing that the four DELs AC145124.1, AC010168.2, MIR200CHG, and AC098613.1 can collectively be an independent prognostic indicator for BLCA patients.

DEGs from the high-and low-risk groups were enriched in different diseases, contributing to the differential prognosis of patients in high- and low-risk groups. We noticed that when patients with BLCA were separated into low- and high-risk groups based on their four DELsrisk score, their DEGs and related signaling pathways were distinguishable. For instance, KEGG analysis found the pathway 'vascular smooth muscle contraction' only in the low-risk group, and 'muscular disease, muscular tissue disease, myopathy, cardiomyopathy' was found using DO analysis. In contrast, pathways related to 'hypertrophic cardiomyopathy' was found in the high-risk group by KEGG analysis and 'tetralogy of Fallot, glycogen storage disease and glycogen metabolism disorder' were enriched in DO analysis. To gain a better understanding of these DELs in BLCA, we also searched for protein-coding genes adjacent to the DELs. Fascinatingly, we found the protein-coding gene HIST4H4 68bp upstream of the DELsAC010168.2. Importantly, the clinical value of HIST4H4 in BLCA was observed at both the mRNA and protein levels, and it is positively correlated the expression level of AC010168.2 as shown both RNA-seq data from TCGA and in-house RT-qPCR validation. Hence, we hypothesize that the novel lncRNA AC010168.2 may modulate the expression of the protein-coding gene HIST4H4, or they could collaborate through some unknown interaction to affect the survival of BLCA patients.

LncRNAs are RNAs of more than 200 nucleotides in length, which have no coding structure. At present, several mechanisms of lncRNA activity have been discovered. LncRNAs exert their functions via various mechanisms by binding to DNA, RNA, or proteins at the cellular level. At the transcriptional level, lncRNAs are able to direct the course of correction of the guide chromatin remodeling complexes to the appropriate chromosomal positions, thus regulating the equilibrium between transcriptionally active chromatin and 
silent heterochromatin in both local and global conditions. In addition, IncRNAs are capable of inhibiting or facilitating the recruitment of RNA pol II, transcription factors, and/or cofactors to gene promoters, thus modulating the transcription of their target genes. Posttranscriptionally, lncRNAs have the capacity to adjust alternative splicing of pre-mRNAs and hence display functions of transcriptomic complexity. Additionally, lncRNAs can influence the stability and translation of mRNA via base pairing with mRNA molecules. It has also been well-documented that IncRNAs can act as competing endogenous RNAs (ceRNAs) to participate in the competition for miRNA binding, thus stopping their activity and affecting the translation of miRNA target genes. Furthermore, IncRNA can also be programmed into small, single-, or double-stranded RNAs, which may cause target mRNA degradation, similar to the function of small interfering RNAs. Moreover, the flexible scaffold nature of lncRNAs enables them to combine multiple protein factors that cannot collaborated or interfere through only protein-protein interactions. The scaffold effect of IncRNAs also plays an essential part in protein activity, localization, and subcellular structure formation [42, 43]. In the current study, we identified four survival-associated lncRNAs (AC145124.1, AC010168.2, MIR200CHG, and AC098613.1) from the DELs in TCGA and built a prognostic signature with the four lncRNAs to predict the prognosis of BLCA patients. Among these four lncRNAs, the noteworthy up-regulated expression of AC010168.2, MIR200CHG, and AC098613.1 was observed in tumor than in non-tumor tissue, unlike that of AC145124.1. The up-regulated IncRNAs protective actions may be partially explained by the various functional mechanisms of IncRNAs described above. For example, up-regulated AC010168.2, MIR200CHG, and AC098613.1 in BLCA may act as ceRNAs to competitively bind miRNAs and thereby inhibit the function of miRNAs to enhance the expression of miRNA target genes, which may include tumor suppressors. However, this hypothesis needs to be validated in a series of rigorous experiments.

There are some deficiencies in the current work that need to be addressed. First, the sequencing data from TCGA contained a small control group of only 19 tumor-free adjacent normal bladder tissue samples. Although we attempted to utilize microarray data from GEO, performed in-house RT-qPCR detection, and performed a meta-analysis, the current findings still need to be validated with a larger sample size. Second, we did not perform a prognostic analysis with our own clinical samples due to the lack of sufficient follow-up information. A larger sample size with longer follow-up is required in the future. Third, none of the four IncRNAs identified in the current study have been reported in the literature, and so the functions of these lncRNAs remain entirely unknown. Finally, a series of rigorous experiments is needed to uncover the roles of these four lncRNAs in BLCA.

\section{Conclusion}

In conclusion, four novel IncRNAs (AC145124.1, AC010168.2, MIR200CHG, and AC098613.1) were used to generate a valuable risk score, which can serve as an independent indicator to predict the survival status of BLCA patients due to their influences on distinct signaling pathways. This in silico study may pioneer future exploration into the study of prognostic lncRNAs in BLCA.

\section{Acknowledgements}

The study was supported by the International Communication of Guangxi Medical University Graduate Education (2017), the Project Promoting Basic Capacity for Young and Middle-aged University Teachers in Guangxi (KY2016YB090), the Guangxi Natural Science Fund for Innovation Research Team (2013GXNSFFA019002, 2016GXNSFGA380006), the Science and Technology Development Plan of Guangxi (Guikegong 1355005-3-17), the 


\section{Cellular Physiology Cell Physiol Biochem 2018;50:1474-1495 \begin{tabular}{l|l|l} 
and Biochemistry Published 10.1159/000494647 & $\begin{array}{l}\text { ○ 2018 The Author(s). Published by S. Karger AG, Basel } \\
\text { www.karger.com/cpb }\end{array}$ \\
\hline
\end{tabular}}

He et al.: a Prognostic Four-IncRNA-Based Risk Score For BLCA

Guangxi Natural Science Foundation (2016GXNSFBA380150), National Natural Science Foundation of China (81760454), and the Innovation Project of Guangxi Graduate Education (YCBZ2017044).

\section{Disclosure Statement}

The authors have no conflicts of interest to declare.

\section{References}

1 Kamat AM, Hahn NM, Efstathiou JA, Lerner SP, Malmstrom PU, Choi W, Guo CC, Lotan Y, Kassouf W: Bladder cancer. Lancet 2016;388:2796-2810.

-2 Siegel RL, Miller KD, Jemal A: Cancer Statistics, 2017. CA Cancer J Clin 2017;67:7-30.

-3 Chen W, Zheng R, Baade PD, Zhang S, Zeng H, Bray F, Jemal A, Yu XQ He J: Cancer statistics in China, 2015. CA Cancer J Clin 2016;66:115-132.

4 Luo Y, Zhang X, Mo M, Tan Z, Huang L, Zhou H, Wang C, Wei F, Qiu X, He R, Chen G: High Ki-67 Immunohistochemical Reactivity Correlates With Poor Prognosis in Bladder Carcinoma: A Comprehensive Meta-Analysis with 13, 053 Patients Involved. Medicine (Baltimore) 2016;95:e3337.

5 Huang B, Zhai W, Hu G, Huang C, Xie T, Zhang J, Xu Y: MicroRNA-206 acts as a tumor suppressor in bladder cancer via targeting YRDC. Am J Transl Res 2016;8:4705-4715.

6 Warrick JI, Walter V, Yamashita H, Chung E, Shuman L, Amponsa VO, Zheng Z, Chan W, Whitcomb TL, Yue F, Iyyanki T, Kawasawa YI, Kaag M, Guo W, Raman JD, Park JS, DeGraff DJ: FOXA1, GATA3 and PPAR Cooperate to Drive Luminal Subtype in Bladder Cancer: A Molecular Analysis of Established Human Cell Lines. Sci Rep 2016;6:38531.

-7 Li QQ, Hao JJ, Zhang Z, Hsu I, Liu Y, Tao Z, Lewi K, Metwalli AR, Agarwal PK: Histone deacetylase inhibitorinduced cell death in bladder cancer is associated with chromatin modification and modifying protein expression: A proteomic approach. Int J Oncol 2016;48:2591-2607.

-8 Gao L, Li SH, Tian YX, Zhu QQ Chen G, Pang YY, Hu XH: Role of downregulated miR-133a-3p expression in bladder cancer: a bioinformatics study. Onco Targets Ther 2017;10:3667-3683.

-9 Wei Y, He R, Wu Y, Gan B, Wu P, Qiu X, Lan A, Chen G, Wang Q Lin X, Chen Y, Mo Z: Comprehensive investigation of aberrant microRNA profiling in bladder cancer tissues. Tumour Biol 2016;37:1255512569.

10 Gan X, Lin X, He R, Lin X, Wang H, Yan L, Zhou H, Qin H, Chen G: Prognostic and Clinicopathological Significance of Downregulated p16 Expression in Patients with Bladder Cancer: A Systematic Review and Meta-Analysis. Dis Markers 2016;2016:5259602.

11 Ponting CP, Oliver PL, Reik W: Evolution and functions of long noncoding RNAs. Cell 2009;136:629-641.

12 Wang H, Niu L, Jiang S, Zhai J, Wang P, Kong F, Jin X: Comprehensive analysis of aberrantly expressed profiles of lncRNAs and miRNAs with associated ceRNA network in muscle-invasive bladder cancer. Oncotarget 2016;7:86174-86185.

13 Guo S, Chen W, Luo Y, Ren F, Zhong T, Rong M, Dang Y, Feng Z, Chen G: Clinical implication of long noncoding RNA NEAT1 expression in hepatocellular carcinoma patients. Int J Clin Exp Pathol 2015;8:53955402.

14 Fang Z, Zhang S, Wang Y, Shen S, Wang F, Hao Y, Li Y, Zhang B, Zhou Y, Yang H: Long non-coding RNA MALAT-1 modulates metastatic potential of tongue squamous cell carcinomas partially through the regulation of small proline rich proteins. BMC Cancer 2016;16:706.

15 Jiao C, Song Z, Chen J, Zhong J, Cai W, Tian S, Chen S, Yi Y, Xiao Y: IncRNA-UCA1 enhances cell proliferation through functioning as a ceRNA of Sox4 in esophageal cancer. Oncol Rep 2016;36:2960-2966.

16 Qi D, Li J, Que B, Su J, Li M, Zhang C, Yang M, Zhou G, Ji W: Long non-coding RNA DBCCR1-003 regulate the expression of DBCCR1 via DNMT1 in bladder cancer. Cancer Cell Int 2016;16:81.

17 Zhang Y, He RQ, Dang YW, Zhang XL, Wang X, Huang SN, Huang WT, Jiang MT, Gan XN, Xie Y, Li P, Luo DZ, Chen G, Gan TQ: Comprehensive analysis of the long noncoding RNA HOXA11-AS gene interaction regulatory network in NSCLC cells. Cancer Cell Int 2016;16:89. 


\section{Cellular Physiology Cell Physiol Biochem 2018;50:1474-1495 \begin{tabular}{l|l|l} 
and Biochemistry Published online: 26 October 2018 & $\begin{array}{l}\text { ○ 2018 The Author(s). Published by S. Karger AG, Basel } \\
\text { www.karger.com/cpb }\end{array}$ \\
\hline
\end{tabular}}

18 Duan W, Du L, Jiang X, Wang R, Yan S, Xie Y, Yan K, Wang Q, Wang L, Zhang X, Pan H, Yang Y, Wang C: Identification of a serum circulating lncRNA panel for the diagnosis and recurrence prediction of bladder cancer. Oncotarget 2016;7:78850-78858.

19 Tang R, Jiang M, Liang L, Xiong D, Dang Y, Chen G: Long Noncoding RNA MALAT-1 Can Predict Poor Prognosis: A Meta-Analysis. Med Sci Monit 2016;22:302-309.

-20 Huang Z, Huang L, Shen S, Li J, Lu H, Mo W, Dang Y, Luo D, Chen G, Feng Z: Sp1 cooperates with Sp3 to upregulate MALAT1 expression in human hepatocellular carcinoma. Oncol Rep 2015;34:2403-2412.

-21 Fang Q, Chen X, Zhi X: Long Non-Coding RNA (LncRNA) Urothelial Carcinoma Associated 1 (UCA1) Increases Multi-Drug Resistance of Gastric Cancer via Downregulating miR-27b. Med Sci Monit 2016;22:3506-3513.

-22 Huang M, Zhong Z, Lv M, Shu J, Tian Q, Chen J: Comprehensive analysis of differentially expressed profiles of IncRNAs and circRNAs with associated co-expression and ceRNA networks in bladder carcinoma. Oncotarget 2016;7:47186-47200.

-23 Berrondo C, Flax J, Kucherov V, Siebert A, Osinski T, Rosenberg A, Fucile C, Richheimer S, Beckham CJ: Expression of the Long Non-Coding RNA HOTAIR Correlates with Disease Progression in Bladder Cancer and Is Contained in Bladder Cancer Patient Urinary Exosomes. PLoS One 2016;11:e0147236.

24 Bao Z, Zhang W, Dong D: A potential prognostic lncRNA signature for predicting survival in patients with bladder urothelial carcinoma. Oncotarget 2017;8:10485-10497.

-25 Droop J, Szarvas T, Schulz WA, Niedworok C, Niegisch G, Scheckenbach K, Hoffmann MJ: Diagnostic and prognostic value of long noncoding RNAs as biomarkers in urothelial carcinoma. PLoS One 2017;12:e0176287.

26 Harrow J, Frankish A, Gonzalez JM, Tapanari E, Diekhans M, Kokocinski F, Aken BL, Barrell D, Zadissa A, Searle S, Barnes I, Bignell A, Boychenko V, Hunt T, Kay M, Mukherjee G, Rajan J, Despacio-Reyes G, Saunders G, Steward C et al.: GENCODE: the reference human genome annotation for The ENCODE Project. Genome research 2012;22:1760-1774.

-27 Robinson MD, McCarthy DJ, Smyth GK: edgeR: a Bioconductor package for differential expression analysis of digital gene expression data. Bioinformatics 2010;26:139-140.

28 Zeng JH, Liang L, He RQ, Tang RX, Cai XY, Chen JQ, Luo DZ, Chen G: Comprehensive investigation of a novel differentially expressed lncRNA expression profile signature to assess the survival of patients with colorectal adenocarcinoma. Oncotarget 2017;8:16811-16828.

-29 Tang RX, Chen WJ, He RQ, Zeng JH, Liang L, Li SK, Ma J, Luo DZ, Chen G: Identification of a RNA-Seq based prognostic signature with five IncRNAs for lung squamous cell carcinoma. Oncotarget 2017;8:5076150773.

-30 Li HM, Yang H, Wen DY, Luo YH, Liang CY, Pan DH, Ma W, Chen G, He Y, Chen JQ: Overexpression of LncRNA HOTAIR is Associated with Poor Prognosis in Thyroid Carcinoma: A Study Based on TCGA and GEO Data. Hormone and metabolic research $=$ Hormon- und Stoffwechselforschung $=$ Hormones et metabolisme 2017;49:388-399.

-31 Wang Z, Wu Q, Feng S, Zhao Y, Tao C: Identification of four prognostic LncRNAs for survival prediction of patients with hepatocellular carcinoma. PeerJ 2017;5:e3575.

-32 Zhang ZL, Zhao LJ, Chai L, Zhou SH, Wang F, Wei Y, Xu YP, Zhao P: Seven LncRNA-mRNA based risk score predicts the survival of head and neck squamous cell carcinoma. Sci Rep 2017;7:309.

-33 Adler P, Kolde R, Kull M, Tkachenko A, Peterson H, Reimand J, Vilo J: Mining for coexpression across hundreds of datasets using novel rank aggregation and visualization methods. Genome biology 2009;10:R139.

34 Wang Y, Zhang J, Li L, Xu X, Zhang Y, Teng Z, Wu F: Identification of Molecular Targets for Predicting Colon Adenocarcinoma. Med Sci Monit 2016;22:460-468.

-35 Lv J, Zhu B, Zhang L, Xie Q Zhuo W: Detection and screening of small molecule agents for overcoming Sorafenib resistance of hepatocellular carcinoma: a bioinformatics study. Int J Clin Exp Med 2015;8:23172325.

-36 Zhao L, Zhang J, Tan H, Wang W, Liu Y, Song R, Wang L: Gene function analysis in osteosarcoma based on microarray gene expression profiling. Int J Clin Exp Med 2015;8:10401-10410.

-37 Xu X, Li H: Integrated microRNAgene analysis of coronary artery disease based on miRNA and gene expression profiles. Mol Med Rep 2016;13:3063-3073. 


\section{Cellular Physiology Cell Physiol Biochem 2018;50:1474-1495 and Biochemistry \begin{tabular}{l|l} 
DOl: 10.1159/000494647 & $\begin{array}{l}\text { O 2018 The Author(s). Published by S. Karger AG, Basel } \\
\text { www.karger.com/cpb }\end{array}$ \\
\cline { 1 - 2 }
\end{tabular}

38 Cai Y, Zhong X, Wang Y, Yang J: Screening feature genes of astrocytoma using a combined method of microarray gene expression profiling and bioinformatics analysis. Int J Clin Exp Med 2015;8:18004-18012.

-39 Petrizzo A, Caruso FP, Tagliamonte M, Tornesello ML, Ceccarelli M, Costa V, Aprile M, Esposito R, Ciliberto G, Buonaguro FM, Buonaguro L: Identification and Validation of HCC-specific Gene Transcriptional Signature for Tumor Antigen Discovery. Sci Rep 2016;6:29258.

-40 Han L, Ma P, Liu SM, Zhou X: Circulating long noncoding RNA GAS5 as a potential biomarker in breast cancer for assessing the surgical effects. Tumour Biol 2016;37:6847-6854.

41 Beaver LM, Kuintzle R, Buchanan A, Wiley MW, Glasser ST, Wong CP, Johnson GS, Chang JH, Lohr CV, Williams DE, Dashwood RH, Hendrix DA, Ho E: Long noncoding RNAs and sulforaphane: a target for chemoprevention and suppression of prostate cancer. J Nutr Biochem 2017;42:72-83.

42 Hu W, Alvarez-Dominguez JR, Lodish HF: Regulation of mammalian cell differentiation by long non-coding RNAs. EMBO Rep 2012;13:971-983.

43 Karlsson 0, Baccarelli AA: Environmental Health and Long Non-coding RNAs. Curr Environ Health Rep 2016;3:178-187. 